Supplementary material

\title{
Chemical and toxicological properties of emissions from a light-duty CNG vehicle fueled with renewable natural gas
}

Yin $\mathrm{Li}^{\mathrm{a}}$, Jian Xue ${ }^{\mathrm{a}}$, Joshua Peppers ${ }^{\mathrm{b}}$, Norman Y. Kado ${ }^{\mathrm{c}}$, Christoph F.A. Vogel ${ }^{\mathrm{c}}$, Christopher P.

Alaimo $^{\mathrm{a}}$, Peter G. Green ${ }^{\mathrm{a}}$, Ruihong Zhang ${ }^{\mathrm{b}}$, Bryan M. Jenkins ${ }^{\mathrm{b}}$, Minji Kimª, Thomas M. Young ${ }^{\mathrm{a}}$, and Michael J. Kleeman ${ }^{\mathrm{a}, *}$

a Department of Civil and Environmental Engineering, University of California - Davis, Davis, California 95616, USA

${ }^{\mathrm{b}}$ Department of Biological and Agricultural Engineering, University of California - Davis, Davis, California 95616, USA

${ }^{c}$ Department of Environmental Toxicology and Center for Health and the Environment, University of California - Davis, Davis, California 95616, USA

*corresponding author: mjkleeman@ucdavis.edu

Department of Civil and Environmental Engineering

University of California

Davis, CA 95616

Phone: 5307528386

22 pages

6 Figures

10 Tables 
Biomethane composition analysis

RNG cylinders were transported back to the lab for chemical composition analysis. A dual stage natural gas regulator with stainless steel diaphragm (The Fitting Source, Inc.) was connected to the compressed gas cylinder to reduce the pressure from $3600 \mathrm{psi}$ to less than $0.3 \mathrm{psi}$. From the outlet of the regulator, RNG was sampled with an $8 \mathrm{~L}$ Tedlar ${ }^{\circledR}$ bag (SKC-West, Inc.) for major components and sulfur-containing compounds analysis, and with adsorbent tubes (charcoal tube, DNPH coated silica tube and XAD tube, SKC, Inc.) for hydrocarbons, carbonyls, and a variety of different VOC and SVOCs analysis. The details of sampling and analysis methods as well as a comprehensive list of all the compounds analyzed can be found in previous studies ${ }^{1,2}$.

Engine exhaust toxicity analysis

ROS assay

The macrophage reactive oxygen species (ROS) assay measures the ROS generating capacity of water extracts of particulate matter in exhaust samples. The reagent DCFH-DA (2',7'-Dichlorofluorescin diacetate) is a cell-permeable ROS indicator that is modified by the ubiquitous esterases in the cell cytoplasm and then by the ROS species to generate a fluorescent product DCF (Dichlorofluorescein), which is detected using a fluorometer. Rat alveolar macrophage cells (NR8383) were exposed to filter water extract at three different dilutions for a 2.5 -hour incubation $\left(37^{\circ} \mathrm{C}, 5 \% \mathrm{CO}_{2}\right)$, together with positive controls exposed to Zymosan as well as untreated controls. Linear regression of fluorescence units vs. Zymosan concentration was performed so that final ROS concentrations were expressed in units of equivalent $\mu \mathrm{g}$-Zymosan.

\section{Mutagenicity assay}

The mutagenicity bioassay adopted a micro-suspension modification of the Salmonella/microsome Ames assay described in previous studies ${ }^{3,4}$ that is ten times more sensitive than the standard plate incorporation method. Frame-shift mutations of Salmonella typhimurium tester strain TA98 were observed, with liver homogenate (S9) from male Sprague Dawley rats (Mol Tox, Boone, NC) added to provide metabolic activation of the sample. Filters were stored at $-20^{\circ} \mathrm{C}$ until shipment to the University of California, Davis lab where they were extracted using pressurized solvent dichloromethane (Burdick and Jackson GC grade) at 2,000 psi, $100^{\circ} \mathrm{C}$. The extracts obtained were dried and re-dissolved in dimethyl sulfoxide (DMSO) for testing.

\section{Biomarker assay}

The expression of in-vitro pro-inflammatory markers induced by particulate matter from vehicle exhaust was measured in human U937 macrophage cells (American Tissue Culture Collection, Manassas, VA). Macrophages U937 are the first line of defense in human lungs. Substances in exhaust may interact with the macrophage cells though the Toll-Like Receptors (TLR), Aryl hydrocarbon Receptor (AhR) and the NF-KappaB protein complex to induce inflammatory responses. After treating the U937 macrophage cells for 6 hours with water extract of exhaust PM collecting filter, mRNA was isolated from the cells and reverse-transcribed into cDNA for quantitative expression analysis using qPCR. Results were normalized to housekeeping gene $\beta$-Actin expression and expressed as fold increase of mRNA in treated cells relative to untreated cells. 
Engine exhaust non-target chemical analysis

Engine exhaust samples from the CVS dilution tunnel were drawn through an $8 \times 110 \mathrm{~mm} 400$ $\mathrm{mg} / 200 \mathrm{mg}$ XAD-2 sorbent tube (SKC, Inc.) during the entire UC cycle (1735 seconds) at a flow rate of $1 \mathrm{~L} \mathrm{~min}^{-1}$. Sorbent tubes were unsealed immediately prior to sampling, and flow rate was controlled with a calibrated 1-5 $\mathrm{L} \mathrm{min}^{-1}$ adjustable flow meter (Dwyer Instruments, Inc.). Negative pressure was created at the back end of the sampling apparatus using an explosion-proof Teflon diaphragm pump. At the conclusion of the sampling period, the sorbent tube was immediately capped, labeled, and placed into a cooler. Once transported back to the lab, samples were stored in a $0{ }^{\circ} \mathrm{C}$ freezer for up to 30 days until extraction. Sorbent tubes were extracted by breaking open each section and separately transferring the sorbent material to labeled glass vials. Ethyl acetate $(1 \mathrm{ml})$ was added to each vial, which was then capped and sonicated for 30 minutes. The supernatant liquid was transferred to a labeled amber glass autosampler vial.

Analysis was carried out on an Agilent 7890B Gas Chromatograph and mass spectra were acquired using a 7200B Quadrupole-Time-of-Flight Mass Spectrometer (GC-QTOF-MS). Each sample run includes a system blank, two sample blanks (1 set of sorbent tube extracts), calibration standards, and the samples. A series of standard semivolatile organic compounds (Restek 318508270 Megamix) was used to produce a retention time-retention index calibration curve. Separation was accomplished using an Agilent J\&W HP5-MS UI column ( $30 \mathrm{~m} \times 0.25 \mathrm{~mm} \times 0.25 \mu \mathrm{m})$ with an injection volume of $1.0 \mu \mathrm{l}$ and a flow rate of $0.8 \mathrm{~mL} \mathrm{~min}^{-1}$ in $(\mathrm{He})$. The injector temperature was $250^{\circ} \mathrm{C}$ and the temperature program was: $35^{\circ} \mathrm{C}$ for $3 \mathrm{~min}$, ramp to $325^{\circ} \mathrm{C}$ at $4^{\circ} \mathrm{C} \mathrm{min}^{-1}$, hold at $325^{\circ} \mathrm{C}$ for 3 minutes.

Raw data from the GC-QTOF-MS experiments were first converted from vendor format to analysis base file format for further processing (Reifycs Analysis Base File Converter v. 4.0.0). All data were subsequently deconvoluted and aligned using MS-DIAL (v. 3.66). Compounds not on the target list were tentatively identified based on retention index and mass spectral similarity using the NIST17 database with a cutoff score of 800 . When the aligned nontarget feature was present in the standard solution, the feature was considered to be confirmed. If the nontarget feature was not present in the standard but was independently identified in each sample as the same compound its identification is considered tentative/confident. In some cases, the feature alignment was good but the spectral quality did not allow a confident identification of the compound. These features are listed as tentative/uncertain if at least one sample had an annotation that met the RI and spectral similarity cutoff score (800). If that criterion was not met, the feature is listed as unknown. 
Elastic net regression ${ }^{5}$ on exhaust features and toxicity responses

The Elastic Net Regression is a regularized regression method that linearly combines the L1 and L2 penalties of the lasso and ridge methods. It fits a least-squares regression model to the data, subject to a penalty on a weighted sum of the absolute value of the model coefficients (the L1-norm) and the sum of squares the model coefficients (the L2-norm):

i.e. solve for $\beta$ 's in minimizing:

$$
\sum_{i=1}^{n}(Y-\hat{Y})^{2}+\lambda \sum_{j=1}^{p}\left[\frac{1}{2}(1-\alpha) \beta_{j}^{2}+\alpha\left|\beta_{j}\right|\right]
$$

where

$$
\hat{Y}=\sum_{j=1}^{p} \beta_{j} X_{j}
$$

The penalty was selected using an $\alpha$ value of 0.5 . Cross validation was not used due to a small sample size. In the fitted model, variables with a negligible association with the outcome have a coefficient of 0 (a result of the penalty on the L1-norm) and are thus effectively not included in the model.

Chemical data (emission factors or peak area count of mass spec) were transformed prior to statistical analysis using the relationship $\log _{2}(\mathrm{x}+\mathrm{c})$, where $\mathrm{x}$ is the original value and $\mathrm{c}$ was selected to minimize the absolute value of the regression slope of the analyte standard deviations on the analyte means. The log-transformed data were then normalized with the Cyclic Loess normalization function in Bioconductor limma package (version 3.46.0). The toxicity response data (i.e. ROS, CYP1A1, IL-8, COS-2, mutagenicity) were also $\log _{2}$ transformed. Elastic net regression was then used to identify the linear combination of chemical features that was most predictive of $\log 2$ transformed toxicity response. Positive coefficients were returned for each toxicity end point, with the larger coefficients interpreted as a stronger association.

Fuel consumption calculation ${ }^{6}$ :

$$
\text { Fuel economy }\left(\text { mile } \cdot \mathrm{m}^{-3}\right)=\frac{\text { Density of fuel at } 15^{\circ} \mathrm{C}\left(\mathrm{g} \cdot \mathrm{m}^{-3}\right)}{1.212 * \mathrm{cf} *\left(\mathrm{HC} \times \frac{m_{C}}{m_{\mathrm{HC}}}+\mathrm{CO} \times \frac{m_{C}}{m_{\mathrm{CO}}}+\mathrm{CO}_{2} \times \frac{m_{C}}{m_{\mathrm{CO}_{2}}}\right)}
$$

cf Where,correction factor $\underline{\underline{0}}$

$$
.82 \overline{\overline{5}}+0.0693 \times(\text { fuel } \mathrm{H}: \text { C ratio }) \text {, fuel } \mathrm{H}: \mathrm{C} \text { ratio is } 3.88 \text { for } \mathrm{CNG},
$$

3.96 for RNG1 and 3.99 for RNG2 calculated based on fuel composition measurements summarized in Table 1.

$\frac{m_{C}}{m_{H C}}=\frac{\text { mass of carbon from } H C s \text { in the fuel }}{\text { mass of } H C \text { s in the fuel }}$ calculated from fuel composition is 0.754 for CNG, 0.750 for

RNG1 and 0.749 for RNG2

$\frac{m_{C}}{m_{C O}}=0.429, \frac{m_{C}}{m_{C O_{2}}}=0.273$, for all fuels

$\mathrm{HC}, \mathrm{CO}$ and $\mathrm{CO}_{2}$ are mass emission factors measured from exhaust $\left(\mathrm{g} \cdot \mathrm{mile} \mathrm{e}^{-1}\right)$. 
Fuel economy and $\mathrm{CO}_{2}$ emissions

Figures S2(a) and (b) present fuel economy and $\mathrm{CO}_{2}$ emission factors averaged over the entire cold-start UC cycle and for each phase of the UC cycle. Each bar represents the average emissions from two tests of the indicated fuel type, while error bars represent the range (min and max values). The cold start effect and the amount of acceleration within the speed trace influence the fuel economy and $\mathrm{CO}_{2}$ emission during different phases of the UC cycle. Phase 1 and 3 emission can be compared to quantify the effect of the cold start (Phase 1) vs. hot start (Phase 2) engine conditions over an identical $300 \mathrm{sec}$ driving pattern. Phase 2 was based on a longer $1135 \mathrm{sec}$ high-speed driving period designed to represent highway travel. The fuel economy was lowest in phase 1 and highest in phase 2, with an overall cycleaveraged value similar to phase 2 . The fuel economy of the test vehicle was highest when using CNG (2.87 miles $\mathrm{m}^{-3}$ ) with reduced fuel economy measured for RNG1 (-3.1\%) and RNG2 (-4.9\%). This trend is consistent with the fact that $\mathrm{CNG}\left(38.27 \mathrm{MJ} \cdot \mathrm{m}^{-3}\right)$ had the highest fuel energy content with lower energy content for RNG1 (-5.1\% vs. CNG) and RNG2 (-7.3\% vs. CNG). The energy consumed for each mile traveled showed less variability between CNG (13.3 MJ.mile $\left.{ }^{-1}\right)$, RNG1 (13.1 MJ.mile ${ }^{-1} ;-1.5 \%$ vs. CNG), and RNG2 (12.9 MJ.mile ${ }^{-1} ;-3 \%$ vs. $\left.\mathrm{CNG}\right) . \mathrm{CO}_{2}$ emissions factors were inversely correlated with fuel economy, with the lowest values measured when using CNG $\left(682 \mathrm{~g} \mathrm{mile}^{-1}\right)$ and slightly higher values measured for RNG1 (+1.2\%) and RNG2 (+5.0\%). $\mathrm{CO}_{2}$ emissions were higher from RNG because the lower heating value of RNG required more fuel consumption per mile, and because RNG had higher fuel $\mathrm{CO}_{2}$ content, as previously discussed. Karavalakis et al., $2012^{7}$ used the UC driving cycle to test two passenger cars (2002 Ford Crown Victoria and 2006 Honda Civic GX) fueled with CNG yielding slightly higher fuel economy and lower pollutant emissions rates than the current study. This result is expected since the current study tested a light duty cargo van rather than a light duty passenger car.

Carbon monoxide and unburned HCs emissions

$\mathrm{CO}$ emissions reflect the combustion and oxidation conditions inside the vehicle engine and exhaust system. Figure S2(c) shows that phase 1 of the UC cycle had the highest $\mathrm{CO}$ emissions because the temperature of both engine and three-way catalyst (TWC) were initially low yielding reduced oxidation of $\mathrm{CO}$ to $\mathrm{CO}_{2}$. The cold-start effect is also visible from the time series plot of $\mathrm{CO}$ concentration in undiluted exhaust measured by FTIR as shown in Figure S3(a). CO concentration quickly increased as the vehicle started moving and then decreased after $\sim 100$ seconds as the engine and TWC warmed up. Emissions are generally ranked in descending order of phase $1>$ phase $2>$ phase 3 because phase 1 contains the cold-start effect, while phase 2 consists of several fast-acceleration periods to reach a maximum speed of $107 \mathrm{~km} \cdot \mathrm{hr}^{-1}$. The demand for increased speed and power required the engine to operate at a rich air/fuel $(\mathrm{A} / \mathrm{F})$ ratio $(\lambda<1$ where $\lambda=$ actual $\mathrm{A} / \mathrm{F}$ ratio $\div$ stoichiometric $\mathrm{A} / \mathrm{F}$ ratio), resulting in higher $\mathrm{CO}$ emission. Overall, $\mathrm{CO}$ emission factors were lowest when using $\mathrm{CNG}$ $\left(0.94 \mathrm{~g} \cdot \mathrm{mile}^{-1}\right)$, with increasing CO emissions when using RNG1 (+25\% vs. CNG) and RNG2 (+59\% vs. $\mathrm{CNG})$. This trend may be caused by increasing fuel $\mathrm{CO}_{2}$ concentrations in the RNG blends. $\mathrm{CO}_{2}$ in the fuel displaces oxygen in the combustion chamber and can act as an inert diluent contributing to pockets of incomplete combustion. This trend was consistent and significant for the phase 2 and phase $3 \mathrm{CO}$ emissions. $\mathrm{CO}$ emissions in phase 1 had higher variability with no clear trend by fuel type.

Figures S2(d) and 1(e) show that emissions of unburned HCs largely follow the CO trend because both constituents result from incomplete fuel combustion and poor oxidation in the catalyst before it 
reaches operating temperatures ${ }^{6}$. The RNG1 and RNG2 emissions of total HCs exceed the CNG HC emissions by $63 \%$ and $72 \%$, respectively. The elevated $\mathrm{HC}$ emissions from RNG result from higher emissions of unburned methane (Figure S2 (d)). CNG emitted higher concentrations of C2 and C3 HCs (ethane and propane) as shown in Figure S2 (e) and Figure S3 (b) and (c), but this increase was overwhelmed by the increased methane emission from RNGs.

Oxides of Nitrogen (NOx), nitrous oxide, and ammonia emissions

High temperature and long residence time favor the formation of thermal $\mathrm{NO}$ xcreated from the $\mathrm{N}_{2}$ in the combustion air. Figure S2(f) shows that the NO xemissions vary significantly from phase to phase with no consistent trend among different fuels. Averaged across the UC cycle, RNG emitted more NOx than CNG in the current study, which is consistent with the results reported by Subramanian et al.6. Both nitrous oxide $\left(\mathrm{N}_{2} \mathrm{O}\right.$, a strong $\left.\mathrm{GHG}\right)$ and ammonia $\left(\mathrm{NH}_{3}\right.$, an important $\mathrm{PM}$ precursor) are formed during TWC reduction of NO in the exhaust8-10. $\mathrm{N}_{2} \mathrm{O}$ is formed on the cold surface of the TWC usually under a narrow equivalent $\lambda$ window of lean $\mathrm{A} / \mathrm{F}$ ratio, while $\mathrm{NH}$ is formed on the hot TWC under a wider $\lambda$ range 8 . The time-series relationship between $\mathrm{NOx}, \mathrm{N}_{2} \mathrm{O}$ and $\mathrm{NH}$ 3for the first 150 seconds of each test is shown in Figures S3(d)-(f). NO x(mostly NO) concentrations quickly increased as the vehicle started, while $\mathrm{N}_{2} \mathrm{O}$ concentrations increased later in the cycle. As the catalyst temperature increased, $\mathrm{NO}$ xand $\mathrm{N}_{2} \mathrm{O}$ concentrations decreased while $\mathrm{NH}$ sincreased. Emission factors for $\mathrm{N}_{2} \mathrm{O}$ from different phases of the UC cycle are presented in Figure S2(g). The cold-start phase 1 emissions were more than 5 times higher than phase 2 and 3 emissions. Fuel composition did not affect $\mathrm{N}_{2} \mathrm{O}$ emissions.

Particulate matter emissions and size distributions

Figure S4 presents the time series of PM mass $\left(\mu \mathrm{g} \cdot \mathrm{m}^{-3}\right)$ and PM number $\left(\# \cdot \mathrm{cm}^{-3}\right)$ concentration measured from the CVS tunnel during RNG2 tests. Emissions from other fuels follow similar trends, with both PM number and mass concentrations increasing with vehicle speed and hard accelerations. The measured size distributions of particle number (Fig S5b) show that the majority of the particles have diameter smaller than $20 \mathrm{~nm}$. This agrees well with previous studies ${ }^{11,12}$, although one study by Lim et al. ${ }^{13}$ reported bimodal distribution with nucleation mode $<10 \mathrm{~nm}$ and accumulation mode $\sim 30$ $\mathrm{nm}$. The calculated size distributions of ultrafine particle $\left(\mathrm{PM}_{0.1}\right)$ mass (Fig S5a) have a peak approaching $100 \mathrm{~nm}$ particle diameter. Particles with mobility diameter greater than $100 \mathrm{~nm}$ were not included in the analysis due to the potential for electrometer noise in the higher channels of the EEPS instrument ${ }^{14}$ and because previous studies found that $\mathrm{CNG}$ engines emit only minor concentrations of particles larger than $100 \mathrm{~nm}^{11,13-16}$. 
Table S1. Experimentally determined limit of detection (LOD) for different compounds measured by FTIR

\begin{tabular}{|c|c|c|}
\hline Compound & LOD & unit \\
\hline $\mathrm{H}_{2} \mathrm{O}$ & 2.72 & $\%$ \\
\hline $\mathrm{CO}_{2}$ & 0.65 & $\%$ \\
\hline $\mathrm{CO}$ & 3.59 & ppm \\
\hline $\mathrm{NO}$ & 0.82 & ppm \\
\hline $\mathrm{NO}_{2}$ & 0.42 & ppm \\
\hline $\mathrm{NO}_{\mathrm{X}}$ & 1.01 & ppm \\
\hline $\mathrm{N}_{2} \mathrm{O}$ & 0.62 & ppm \\
\hline $\mathrm{NH}_{3}$ & 1.18 & ppm \\
\hline $\mathrm{SO}_{2}$ & 1.69 & ppm \\
\hline $\mathrm{CH}_{4}$ & 3.72 & ppm \\
\hline $\mathrm{C}_{2} \mathrm{H}_{6}$ & 1.78 & ppm \\
\hline $\mathrm{C}_{2} \mathrm{H}_{2}$ & 4.05 & ppm \\
\hline $\mathrm{C}_{2} \mathrm{H}_{4}$ & 0.39 & ppm \\
\hline $\mathrm{C}_{3} \mathrm{H}_{6}$ & 1.97 & ppm \\
\hline $\mathrm{C}_{4} \mathrm{H}_{6}$ & 0.81 & ppm \\
\hline $\mathrm{HCHO}_{\mathrm{CHO}}$ & 0.67 & ppm \\
\hline $\mathrm{CH}_{3} \mathrm{OH}$ & 0.36 & ppm \\
\hline
\end{tabular}

Table S2. Experimentally determined limit of detection (LOD) for different compounds measured by PTR-MS

\begin{tabular}{|cc|cc|}
\hline Compound & LOD $(\mathrm{ppb})$ & Compound & LOD $(\mathrm{ppb})$ \\
\hline methanol & 25.4 & ethenone(ketene) & 4.2 \\
ethanol & 38.0 & methyl ketene & 2.1 \\
propanol & 7.4 & propene & 3.5 \\
formaldehyde & 17.7 & butene & 2.5 \\
acetaldehyde & 19.3 & isoprene & 1.0 \\
acetone & 6.9 & butenone & 1.5 \\
formic acid & 20.5 & benzene & 1.0 \\
acetic acid & 14.3 & toluene & 0.9 \\
Propionic acid & 2.6 & ethylbenzene/xylene & 0.9 \\
dimethyl sulfide & 2.9 & propyl benzene & 1.4 \\
acetonitrile & 5.6 & naphthalene & 0.5 \\
& & benzaldehyde & 0.7 \\
\hline
\end{tabular}


Table S3. Concentration (\%) of major compounds measured in different fuels. Limit of quantifications (LOQs), average values as well as standard deviations from 3 measurements are reported. Note that values below LOQs are possible because RNG1 and RNG2 are blends from CNG and different biomethane sources which can each have measured values above or below LOQs.

\begin{tabular}{|c|c|c|c|c|c|c|c|c|c|c|}
\hline Compound Name & LOQ (\%) & \multicolumn{3}{|c|}{$\mathrm{CNG}$} & \multicolumn{3}{|c|}{ RNG 1} & \multicolumn{3}{|c|}{ RNG 2} \\
\hline $\begin{array}{c}\text { Nitrogen/Carbon } \\
\text { Monoxide }\end{array}$ & 0.23 & $1.83 \%$ & \pm & $0.65 \%$ & $2.64 \%$ & \pm & $1.01 \%$ & $1.77 \%$ & \pm & $0.48 \%$ \\
\hline Oxygen/Argon & 0.14 & $0.42 \%$ & \pm & $0.30 \%$ & $0.42 \%$ & \pm & $0.11 \%$ & $0.18 \%$ & \pm & $0.04 \%$ \\
\hline Methane & 0.76 & $91.20 \%$ & \pm & $0.82 \%$ & $93.50 \%$ & \pm & $1.26 \%$ & $93.33 \%$ & \pm & $0.59 \%$ \\
\hline Carbon Dioxide & 0.72 & $0.82 \%$ & \pm & $0.09 \%$ & $1.85 \%$ & \pm & $0.39 \%$ & $4.28 \%$ & \pm & $0.18 \%$ \\
\hline Ammonia & 0.00001 & \multicolumn{3}{|c|}{$\mathrm{ND}^{*}$} & \multicolumn{3}{|c|}{ ND } & \multicolumn{3}{|c|}{ ND } \\
\hline Ethane & 1.29 & $5.41 \%$ & \pm & $0.18 \%$ & $1.50 \%$ & \pm & $0.05 \%$ & $0.42 \%$ & \pm & $0.01 \%$ \\
\hline Ethene & 1.08 & \multicolumn{3}{|c|}{ ND } & \multicolumn{3}{|c|}{ ND } & \multicolumn{3}{|c|}{ ND } \\
\hline Ethyne & 1.07 & \multicolumn{3}{|c|}{ ND } & \multicolumn{3}{|c|}{ ND } & \multicolumn{3}{|c|}{ ND } \\
\hline Propane & 0.23 & $0.33 \%$ & \pm & $0.06 \%$ & $0.09 \%$ & \pm & $0.02 \%$ & $0.03 \%$ & \pm & $0.00 \%$ \\
\hline Propene & 1.07 & \multicolumn{3}{|c|}{ ND } & \multicolumn{3}{|c|}{ ND } & \multicolumn{3}{|c|}{ ND } \\
\hline Propadiene & 0.97 & \multicolumn{3}{|c|}{ ND } & \multicolumn{3}{|c|}{ ND } & \multicolumn{3}{|c|}{ ND } \\
\hline Propyne & 0.97 & \multicolumn{3}{|c|}{ ND } & \multicolumn{3}{|c|}{ ND } & \multicolumn{3}{|c|}{ ND } \\
\hline i-Butane & 0.98 & \multicolumn{3}{|c|}{ ND } & \multicolumn{3}{|c|}{ ND } & \multicolumn{3}{|c|}{ ND } \\
\hline n-Butane & 0.77 & \multicolumn{3}{|c|}{ ND } & \multicolumn{3}{|c|}{ ND } & \multicolumn{3}{|c|}{ ND } \\
\hline 1-Butene & 0.86 & \multicolumn{3}{|c|}{ ND } & \multicolumn{3}{|c|}{ ND } & \multicolumn{3}{|c|}{ ND } \\
\hline i-Butene & 0.85 & \multicolumn{3}{|c|}{ ND } & & $\mathrm{ND}$ & & & ND & \\
\hline trans-2-Butene & 0.72 & & ND & & & ND & & & ND & \\
\hline cis-2-Butene & 0.72 & & ND & & & $\mathrm{ND}$ & & & ND & \\
\hline 1,3-Butadiene & 0.71 & & ND & & & $\mathrm{ND}$ & & & $\mathrm{ND}$ & \\
\hline Isoprene & 0.72 & & ND & & & $\mathrm{ND}$ & & & $\mathrm{ND}$ & \\
\hline i-Pentane & 0.54 & & $\mathrm{ND}$ & & & $\mathrm{ND}$ & & & $\mathrm{ND}$ & \\
\hline n-Pentane & 0.54 & & ND & & & $\mathrm{ND}$ & & & $\mathrm{ND}$ & \\
\hline neo-Pentane & 0.54 & & ND & & & ND & & & ND & \\
\hline Pentenes & 0.54 & & ND & & & $\mathrm{ND}$ & & & ND & \\
\hline
\end{tabular}

${ }^{*} \mathrm{ND}=$ Not Detected. 
Table S4. Concentration (ppbv) of different sulfur-containing compounds measured in different fuels. Limit of quantifications (LOQs), average values as well as standard deviations from 3 measurements are reported. Note that values below LOQs are possible because RNG1 and RNG2 are blends from CNG and different biomethane sources which can each have measured values above or below LOQs.

\begin{tabular}{|c|c|c|c|c|c|c|c|c|c|c|}
\hline Compound name & $\begin{array}{l}\text { LOQ } \\
(\mathrm{ppbv})\end{array}$ & \multicolumn{3}{|c|}{$\mathrm{CNG}$} & \multicolumn{3}{|c|}{ RNG 1} & \multicolumn{3}{|c|}{ RNG 2} \\
\hline Hydrogen Sulfide & 298.7 & $<\mathrm{LOQ}$ & \pm & - & $<\mathrm{LOQ}$ & \pm & - & $<\mathrm{LOQ}$ & \pm & - \\
\hline Sulfur Dioxide & 26.2 & $<$ LOQ & \pm & - & $<$ LOQ & \pm & - & 124.3 & \pm & 30.1 \\
\hline Carbonyl sulfide & 15.2 & $<$ LOQ & \pm & - & 5.1 & \pm & 10.3 & 10.6 & \pm & 5.2 \\
\hline Carbon disulfide & 2.9 & 6.9 & \pm & 4.6 & 53.7 & \pm & 37.2 & 49.3 & \pm & 17.9 \\
\hline Methyl mercaptan & 52.5 & $<\mathrm{LOQ}$ & \pm & - & 11.2 & \pm & 22.4 & 135.2 & \pm & 29.2 \\
\hline Ethyl mercaptan & 7.8 & 171.8 & \pm & 25.4 & 47.8 & \pm & 7.1 & 27.5 & \pm & 24.7 \\
\hline Isopropyl mercaptan & 4.9 & 43.7 & \pm & 16.6 & 110.6 & \pm & 196.9 & 118.8 & \pm & 111.5 \\
\hline n-Propyl mercaptan & 4.3 & 12.0 & \pm & 10.4 & 23.0 & \pm & 39.4 & 31.5 & \pm & 30.6 \\
\hline t-Butyl mercaptan & 4.4 & 677.0 & \pm & 173.1 & 188.2 & \pm & 48.1 & 100.7 & \pm & 66.4 \\
\hline sec-Butyl mercaptan & 4.9 & $<\mathrm{LOQ}$ & \pm & - & 192.3 & \pm & - & 106.9 & \pm & - \\
\hline Dimethyl sulfide & 4.3 & $<\mathrm{LOQ}$ & \pm & - & 756.1 & \pm & 476.9 & 810.6 & \pm & 315.2 \\
\hline Methyl Ethyl sulfide & 3.5 & 5.2 & \pm & 0.6 & 14.3 & \pm & 9.3 & 26.8 & \pm & 17.0 \\
\hline Diethyl sulfide & 5.7 & 28.3 & \pm & 0.2 & 7.9 & \pm & 0.0 & 2.2 & \pm & 0.0 \\
\hline Di-tert-butyl sulfide & 3.0 & $<$ LOQ & \pm & - & $<$ LOQ & \pm & - & $<$ LOQ & \pm & - \\
\hline Dimethyl Disulfide & 0.8 & $<\mathrm{LOQ}$ & \pm & - & 35.0 & \pm & 69.9 & 53.0 & \pm & 34.8 \\
\hline Diethyl Disulfide & 1.1 & $<\mathrm{LOQ}$ & \pm & - & 2.3 & \pm & 1.6 & 1.1 & \pm & 0.8 \\
\hline Thiofuran & 3.0 & $<$ LOQ & \pm & - & $<$ LOQ & \pm & - & 9.7 & \pm & 5.0 \\
\hline Methyl Ethyl Disulfide & 10.0 & $<\mathrm{LOQ}$ & \pm & - & 24.3 & \pm & 30.1 & 30.1 & \pm & 16.7 \\
\hline Methyl i-Propyl Disulfide & 10.0 & 12.2 & \pm & 21.2 & 76.1 & \pm & 117.0 & 70.1 & \pm & 57.4 \\
\hline $\begin{array}{l}\text { Methyl n-Propyl } \\
\text { Disulfide }\end{array}$ & 10.0 & 15.5 & \pm & 0.0 & 34.7 & \pm & 29.3 & 39.6 & \pm & 16.5 \\
\hline Methyl t-Butyl Disulfide & 10.0 & 51.3 & \pm & 11.9 & 139.1 & \pm & 44.5 & 63.4 & \pm & 21.2 \\
\hline Ethyl i-Propyl Disulfide & 10.0 & $<\mathrm{LOQ}$ & \pm & - & $<$ LOQ & \pm & - & 0.0 & \pm & 0.0 \\
\hline Ethyl n-Propyl Disulfide & 10.0 & $<\mathrm{LOQ}$ & \pm & - & 126.4 & \pm & 93.9 & 60.2 & \pm & 44.7 \\
\hline Ethyl t-Butyl Disulfide & 10.0 & 13.6 & \pm & - & 3.8 & \pm & 0.0 & 1.0 & \pm & 0.0 \\
\hline Di-i-Propyl Disulfide & 10.0 & $<\mathrm{LOQ}$ & \pm & - & 0.0 & \pm & 0.0 & 0.0 & \pm & 0.0 \\
\hline $\begin{array}{l}\text { i-Propyl n-Propyl } \\
\text { Disulfide }\end{array}$ & 10.0 & 28.9 & \pm & 35.3 & 17.6 & \pm & 21.6 & 6.8 & \pm & 9.5 \\
\hline Di-n-Propyl Disulfide & 10.0 & 10.7 & \pm & - & 3.0 & \pm & 0.0 & 0.8 & \pm & 0.0 \\
\hline i-Propyl t-Butyl Disulfide & 10.0 & $<$ LOQ & \pm & - & 0.0 & \pm & 0.0 & 0.0 & \pm & 0.0 \\
\hline n-Propyl t-Butyl Disulfide & 10.0 & $<\mathrm{LOQ}$ & \pm & - & 0.0 & \pm & 0.0 & 0.0 & \pm & 0.0 \\
\hline Di-t-Butyl Disulfide & 10.0 & 17.1 & \pm & 5.1 & 31.5 & \pm & 19.9 & 14.1 & \pm & 9.4 \\
\hline Thiophene & 10.0 & 13.0 & \pm & 2.0 & 3.6 & \pm & 0.6 & 14.0 & \pm & 1.8 \\
\hline C1-Thiophenes & 10.0 & $<$ LOQ & \pm & - & 42.5 & \pm & 0.0 & 48.1 & \pm & 8.5 \\
\hline Thiophane & 10.0 & $<\mathrm{LOQ}$ & \pm & - & 1.1 & \pm & 0.9 & 0.5 & \pm & 0.4 \\
\hline Thiophenol & 10.0 & $<\mathrm{LOQ}$ & \pm & - & 1.1 & \pm & 0.9 & 0.5 & \pm & 0.4 \\
\hline
\end{tabular}


Table S5. Concentration (ppbv) of different hydrocarbons measured in different fuels. Limit of quantifications (LOQs), average values as well as standard deviations from 3 measurements are reported. Note that values below LOQs are possible because RNG1 and RNG2 are blends from CNG and different biomethane sources which can each have measured values above or below LOQs.

\begin{tabular}{|c|c|c|c|c|c|c|c|c|c|c|}
\hline & & \multicolumn{3}{|c|}{$\mathrm{CNG}$} & \multicolumn{3}{|c|}{ RNG1 } & \multicolumn{3}{|c|}{ RNG2 } \\
\hline & $\begin{array}{c}\text { LOQ } \\
\text { (ppbv) }\end{array}$ & avg & & std & avg & & std & avg & & std \\
\hline Cyclopentane & 1.9 & $<\mathrm{LOQ}$ & \pm & - & 483.5 & \pm & 372.0 & 233.1 & \pm & 177.3 \\
\hline Methylcyclopentane & 1.9 & $<\mathrm{LOQ}$ & \pm & - & 1555.7 & \pm & 1123.8 & 764.0 & \pm & 535.5 \\
\hline Cyclohexane & 1.9 & $<$ LOQ & \pm & - & 1356.4 & \pm & 963.4 & 664.7 & \pm & 459.1 \\
\hline Methylcyclohexane & 1.9 & 2939.4 & \pm & 46.1 & 841.0 & \pm & 12.8 & 261.4 & \pm & 6.7 \\
\hline Hexanes & 1.9 & 2828.7 & \pm & 587.9 & 2728.8 & \pm & 1384.3 & 1184.6 & \pm & 657.1 \\
\hline Heptanes & 1.9 & 1043.9 & \pm & 48.9 & 1184.1 & \pm & 602.9 & 533.9 & \pm & 287.5 \\
\hline 2,2,4-Trimethylpentane & 1.9 & 36.2 & \pm & 3.5 & 200.2 & \pm & 1.0 & 877.6 & \pm & 118.9 \\
\hline Octanes & 1.9 & $<$ LOQ & \pm & - & 1024.2 & \pm & 593.6 & 559.4 & \pm & 283.9 \\
\hline Nonanes & 0.03 & 145.3 & \pm & 35.7 & 42.9 & \pm & 9.9 & 17.0 & \pm & 3.2 \\
\hline Decanes & 0.003 & 37.2 & \pm & 5.8 & 11.6 & \pm & 1.6 & 5.3 & \pm & 0.6 \\
\hline Undecanes & 0.012 & 8.3 & \pm & 3.2 & 3.4 & \pm & 0.9 & 2.3 & \pm & 0.3 \\
\hline Dodecanes & 0.002 & 3.0 & \pm & 1.0 & 1.3 & \pm & 0.3 & 0.9 & \pm & 0.1 \\
\hline Tridecanes & 0.002 & 0.6 & \pm & 0.3 & 0.3 & \pm & 0.1 & 0.2 & \pm & 0.0 \\
\hline Tetradecanes & 0.009 & $<$ LOQ & \pm & - & 0.0 & \pm & 0.0 & 0.0 & \pm & 0.0 \\
\hline 1,3-Butadiene & 1.0 & $<\mathrm{LOQ}$ & \pm & - & 5.3 & \pm & 0.0 & 10.1 & \pm & 0.1 \\
\hline Benzene & 8.5 & 684.3 & \pm & 230.2 & 715.6 & \pm & 64.0 & 416.7 & \pm & 85.3 \\
\hline Toluene & 4.1 & 768.1 & \pm & 64.4 & 352.6 & \pm & 17.9 & 328.7 & \pm & 17.8 \\
\hline Ethylbenzene & 3.4 & 40.9 & \pm & 0.1 & 68.5 & \pm & 0.0 & 53.7 & \pm & 4.0 \\
\hline m,p-Xylene & 3.1 & 244.9 & \pm & 6.4 & 311.4 & \pm & 1.8 & 204.6 & \pm & 32.2 \\
\hline o-Xylene & 3.0 & 39.6 & \pm & 2.4 & 68.7 & \pm & 0.7 & 57.3 & \pm & 5.2 \\
\hline 1,2,4-Trimethyl benzene & 0.008 & 0.9 & \pm & 0.1 & 0.4 & \pm & 0.0 & 0.6 & \pm & 0.0 \\
\hline 2-Ethyl toluene & 0.003 & 0.3 & \pm & 0.1 & 0.1 & \pm & 0.0 & 0.1 & \pm & 0.0 \\
\hline Isopropyl Benzene & 0.003 & 1.6 & \pm & 0.4 & 0.7 & \pm & 0.1 & 0.7 & \pm & 0.2 \\
\hline Styrene & 2.8 & $<$ LOQ & \pm & & 2.7 & \pm & 0.0 & 3.3 & \pm & 0.0 \\
\hline Isopropylbenzene & 2.8 & 7.7 & \pm & 0.5 & 7.0 & \pm & 0.2 & 6.2 & \pm & 0.3 \\
\hline 4-Ethyltoluene & 1.9 & 22.7 & \pm & 0.6 & 12.5 & \pm & 0.2 & 13.7 & \pm & 0.0 \\
\hline n-Propylbenzene & 2.5 & 14.3 & \pm & 4.7 & 9.9 & \pm & 1.3 & 7.1 & \pm & 1.1 \\
\hline 1,3,5-trimethylbenzene & 2.5 & 11.1 & \pm & 0.9 & 12.5 & \pm & 0.3 & 9.5 & \pm & 0.7 \\
\hline tert-butylbenzene & 2.6 & $<\mathrm{LOQ}$ & \pm & - & 3.3 & \pm & 0.0 & 2.5 & \pm & 0.6 \\
\hline 1,2,4-Trimethylbenzene & 6.1 & $<\mathrm{LOQ}$ & \pm & - & 18.7 & \pm & 0.0 & 20.1 & \pm & 0.0 \\
\hline s-Butylbenzene & 1.5 & $<\mathrm{LOQ}$ & \pm & - & 5.3 & \pm & 0.0 & 6.2 & \pm & 0.5 \\
\hline p-Isopropyltoluene & 472.8 & $<\mathrm{LOQ}$ & \pm & - & 358.4 & \pm & 0.0 & 286.5 & \pm & 0.0 \\
\hline n-butylbenzene & 10.8 & $<\mathrm{LOQ}$ & \pm & - & $<\mathrm{LOQ}$ & \pm & - & $<\mathrm{LOQ}$ & \pm & - \\
\hline Naphthalene & 6.1 & $<\mathrm{LOQ}$ & \pm & - & 30.3 & \pm & 0.0 & 33.1 & \pm & 0.0 \\
\hline
\end{tabular}


Table S6. Concentration (\%) of major compounds measured in different biomethane sources. Limit of quantifications (LOQs), average values as well as standard deviations from 3 measurements are reported.

\begin{tabular}{|c|c|c|c|c|c|c|c|c|c|c|}
\hline Compound Name & LOQ (\%) & \multicolumn{3}{|c|}{ Food waste 1} & \multicolumn{3}{|c|}{ Food waste 2} & \multicolumn{3}{|c|}{ Dairy digester } \\
\hline $\begin{array}{c}\text { Nitrogen/Carbon } \\
\text { Monoxide }\end{array}$ & 0.23 & $2.96 \%$ & \pm & $1.38 \%$ & $0.48 \%$ & \pm & $0 \%$ & $1.85 \%$ & \pm & $0 \%$ \\
\hline Oxygen/Argon & 0.14 & $0.42 \%$ & \pm & $0.10 \%$ & $0 . \%$ & \pm & $0 \%$ & $0 \%$ & \pm & $0 \%$ \\
\hline Methane & 0.76 & $94.39 \%$ & \pm & $1.71 \%$ & $94.43 \%$ & \pm & $0.01 \%$ & $91.00 \%$ & \pm & $0 \%$ \\
\hline Carbon Dioxide & 0.72 & $2.24 \%$ & \pm & $0.53 \%$ & $5.09 \%$ & \pm & $0 \%$ & $7.14 \%$ & \pm & $0 \%$ \\
\hline Ammonia & 0.00001 & \multicolumn{3}{|c|}{$\mathrm{ND}^{*}$} & \multicolumn{3}{|c|}{ ND } & \multicolumn{3}{|c|}{$\mathrm{ND}$} \\
\hline Ethane & 1.29 & \multicolumn{3}{|c|}{ ND } & \multicolumn{3}{|c|}{ ND } & \multicolumn{3}{|c|}{ ND } \\
\hline Ethene & 1.08 & \multicolumn{3}{|c|}{ ND } & \multicolumn{3}{|c|}{ ND } & \multicolumn{3}{|c|}{ ND } \\
\hline Ethyne & 1.07 & \multicolumn{3}{|c|}{ ND } & \multicolumn{3}{|c|}{ ND } & \multicolumn{3}{|c|}{ ND } \\
\hline Propane & 0.23 & \multicolumn{3}{|c|}{ ND } & \multicolumn{3}{|c|}{ ND } & \multicolumn{3}{|c|}{ ND } \\
\hline Propene & 1.07 & \multicolumn{3}{|c|}{ ND } & \multicolumn{3}{|c|}{ ND } & \multicolumn{3}{|c|}{ ND } \\
\hline Propadiene & 0.97 & \multicolumn{3}{|c|}{ ND } & \multicolumn{3}{|c|}{ ND } & \multicolumn{3}{|c|}{ ND } \\
\hline Propyne & 0.97 & \multicolumn{3}{|c|}{ ND } & \multicolumn{3}{|c|}{ ND } & \multicolumn{3}{|c|}{ ND } \\
\hline i-Butane & 0.98 & \multicolumn{3}{|c|}{ ND } & \multicolumn{3}{|c|}{ ND } & \multicolumn{3}{|c|}{ ND } \\
\hline n-Butane & 0.77 & \multicolumn{3}{|c|}{ ND } & & ND & & & ND & \\
\hline 1-Butene & 0.86 & & ND & & & ND & & & ND & \\
\hline i-Butene & 0.85 & & ND & & & ND & & & ND & \\
\hline trans-2-Butene & 0.72 & & ND & & & ND & & & ND & \\
\hline cis-2-Butene & 0.72 & & ND & & & ND & & & ND & \\
\hline 1,3-Butadiene & 0.71 & & ND & & & ND & & & ND & \\
\hline Isoprene & 0.72 & & ND & & & ND & & & ND & \\
\hline i-Pentane & 0.54 & & ND & & & ND & & & ND & \\
\hline n-Pentane & 0.54 & & ND & & & ND & & & ND & \\
\hline neo-Pentane & 0.54 & & ND & & & ND & & & ND & \\
\hline Pentenes & 0.54 & & ND & & & ND & & & ND & \\
\hline
\end{tabular}

${ }^{*} \mathrm{ND}=$ Not Detected. 
Table S7. Concentration (ppbv) of different sulfur-containing compounds measured in different biomethane streams. Limit of quantifications (LOQs), average values as well as standard deviations from three measurements are reported.

\begin{tabular}{|c|c|c|c|c|c|c|c|c|c|c|}
\hline \multirow{2}{*}{$\begin{array}{l}\text { Compound name } \\
\text { Hydrogen Sulfide }\end{array}$} & \multirow{2}{*}{$\begin{array}{c}\begin{array}{c}\text { LOQ } \\
\text { (ppbv) }\end{array} \\
298.7 \\
\end{array}$} & \multicolumn{3}{|c|}{ Food waste 1} & \multicolumn{3}{|c|}{ Food waste 2} & \multicolumn{3}{|c|}{ Dairy digester } \\
\hline & & $<\mathrm{LOQ}$ & \pm & - & $<\mathrm{LOQ}$ & \pm & - & $<\mathrm{LOQ}$ & \pm & - \\
\hline Sulfur Dioxide & 26.2 & $<\mathrm{LOQ}$ & \pm & - & 263.1 & \pm & 87.8 & 148.0 & \pm & 25.6 \\
\hline Carbonyl sulfide & 15.2 & 7.1 & \pm & 14.2 & 24.3 & \pm & 5.3 & $<\mathrm{LOQ}$ & \pm & - \\
\hline Carbon disulfide & 2.9 & 71.8 & \pm & 51.5 & 65.2 & \pm & 5.5 & 9.3 & \pm & 4.8 \\
\hline Methyl mercaptan & 52.5 & 15.5 & \pm & 31.0 & 387.7 & \pm & 81.1 & $<\mathrm{LOQ}$ & \pm & - \\
\hline Ethyl mercaptan & 7.8 & $<\mathrm{LOQ}$ & \pm & - & 42.5 & \pm & 73.6 & $<\mathrm{LOQ}$ & \pm & - \\
\hline Isopropyl mercaptan & 4.9 & 136.3 & \pm & 272.7 & 204.7 & \pm & 180.0 & $<\mathrm{LOQ}$ & \pm & - \\
\hline n-Propyl mercaptan & 4.3 & 27.2 & \pm & 54.4 & 63.3 & \pm & 72.2 & $<\mathrm{LOQ}$ & \pm & - \\
\hline t-Butyl mercaptan & 4.4 & $<\mathrm{LOQ}$ & \pm & - & 145.1 & \pm & 194.1 & $<\mathrm{LOQ}$ & \pm & - \\
\hline sec-Butyl mercaptan & 4.9 & 266.3 & \pm & - & 45.7 & \pm & - & $<\mathrm{LOQ}$ & \pm & - \\
\hline Dimethyl sulfide & 4.3 & 1047.2 & \pm & 660.5 & 1257.3 & \pm & 651.4 & 119.5 & \pm & 40.1 \\
\hline Methyl Ethyl sulfide & 3.5 & 17.8 & \pm & 12.8 & 49.9 & \pm & 48.2 & 14.6 & \pm & 12.3 \\
\hline Diethyl sulfide & 5.7 & $<\mathrm{LOQ}$ & \pm & - & $<\mathrm{LOQ}$ & \pm & - & $<\mathrm{LOQ}$ & \pm & - \\
\hline Di-tert-butyl sulfide & 3.0 & $<\mathrm{LOQ}$ & \pm & - & $<\mathrm{LOQ}$ & \pm & - & $<\mathrm{LOQ}$ & \pm & - \\
\hline Dimethyl Disulfide & 0.8 & 48.4 & \pm & 96.8 & 44.2 & \pm & 14.6 & 88.2 & \pm & 36.6 \\
\hline Diethyl Disulfide & 1.1 & 3.3 & \pm & 2.2 & $<\mathrm{LOQ}$ & \pm & - & $<\mathrm{LOQ}$ & \pm & - \\
\hline Thiofuran & 3.0 & $<\mathrm{LOQ}$ & \pm & - & 14.4 & \pm & 14.0 & 19.9 & \pm & 7.4 \\
\hline Methyl Ethyl Disulfide & 10.0 & 33.7 & \pm & 41.7 & 31.9 & \pm & 20.8 & 31.9 & \pm & 20.0 \\
\hline Methyl i-Propyl Disulfide & 10.0 & 100.7 & \pm & 161.8 & 51.6 & \pm & 26.1 & 70.5 & \pm & 44.3 \\
\hline $\begin{array}{l}\text { Methyl n-Propyl } \\
\text { Disulfide }\end{array}$ & 10.0 & 42.1 & \pm & 40.6 & 26.8 & \pm & 10.1 & 61.3 & \pm & 33.4 \\
\hline Methyl t-Butyl Disulfide & 10.0 & 172.8 & \pm & 61.4 & $<$ LOQ & \pm & - & $<\mathrm{LOQ}$ & \pm & - \\
\hline Ethyl i-Propyl Disulfide & 10.0 & $<\mathrm{LOQ}$ & \pm & - & $<\mathrm{LOQ}$ & \pm & - & $<\mathrm{LOQ}$ & \pm & - \\
\hline Ethyl n-Propyl Disulfide & 10.0 & 175.1 & \pm & 130.1 & $<\mathrm{LOQ}$ & \pm & - & $<\mathrm{LOQ}$ & \pm & - \\
\hline Ethyl t-Butyl Disulfide & 10.0 & $<\mathrm{LOQ}$ & \pm & - & $<\mathrm{LOQ}$ & \pm & - & $<\mathrm{LOQ}$ & \pm & - \\
\hline Di-i-Propyl Disulfide & 10.0 & $<\mathrm{LOQ}$ & \pm & - & $<\mathrm{LOQ}$ & \pm & - & $<\mathrm{LOQ}$ & \pm & - \\
\hline $\begin{array}{l}\text { i-Propyl n-Propyl } \\
\text { Disulfide }\end{array}$ & 10.0 & 13.3 & \pm & 26.6 & $<\mathrm{LOQ}$ & \pm & - & $<\mathrm{LOQ}$ & \pm & - \\
\hline Di-n-Propyl Disulfide & 10.0 & $<\mathrm{LOQ}$ & \pm & - & $<\mathrm{LOQ}$ & \pm & - & $<\mathrm{LOQ}$ & \pm & - \\
\hline i-Propyl t-Butyl Disulfide & 10.0 & $<\mathrm{LOQ}$ & \pm & - & $<\mathrm{LOQ}$ & \pm & - & $<\mathrm{LOQ}$ & \pm & - \\
\hline n-Propyl t-Butyl Disulfide & 10.0 & $<\mathrm{LOQ}$ & \pm & - & $<$ LOQ & \pm & - & $<\mathrm{LOQ}$ & \pm & - \\
\hline Di-t-Butyl Disulfide & 10.0 & 37.1 & \pm & 27.4 & $<\mathrm{LOQ}$ & \pm & - & $<\mathrm{LOQ}$ & \pm & - \\
\hline Thiophene & 10.0 & $<\mathrm{LOQ}$ & \pm & - & 24.9 & \pm & - & 19.0 & \pm & 7.5 \\
\hline C1-Thiophenes & 10.0 & 58.9 & \pm & - & 31.5 & \pm & 16.0 & 70.7 & \pm & 27.3 \\
\hline Thiophane & 10.0 & $<\mathrm{LOQ}$ & \pm & - & $<\mathrm{LOQ}$ & \pm & - & $<\mathrm{LOQ}$ & \pm & - \\
\hline Thiophenol & 10.0 & $<\mathrm{LOQ}$ & \pm & - & $<\mathrm{LOQ}$ & \pm & - & $<\mathrm{LOQ}$ & \pm & - \\
\hline
\end{tabular}


Table S8. Concentration (ppbv) of different hydrocarbons measured in different biomethane streams. Limit of quantifications (LOQs), average values as well as standard deviations from 3 measurements are reported.

\begin{tabular}{|c|c|c|c|c|c|c|c|c|c|c|}
\hline & & \multicolumn{3}{|c|}{ Food waste 1} & \multicolumn{3}{|c|}{ Food waste 2} & \multicolumn{3}{|c|}{ Dairy digester } \\
\hline & $\begin{array}{c}\text { LOQ } \\
\text { (ppbv) }\end{array}$ & avg & & std & avg & & std & avg & & std \\
\hline Cyclopentane & 1.9 & 669.7 & \pm & 515.3 & 8.1 & \pm & 2.1 & $<\mathrm{LOQ}$ & \pm & - \\
\hline Methylcyclopentane & 1.9 & 2154.8 & \pm & 1556.5 & 57.9 & \pm & 22.3 & 13.7 & \pm & - \\
\hline Cyclohexane & 1.9 & 1878.6 & \pm & 1334.4 & 45.4 & \pm & 12.5 & 13.1 & \pm & - \\
\hline Methylcyclohexane & 1.9 & 33.1 & \pm & - & 50.4 & \pm & - & 27.9 & \pm & 23.1 \\
\hline Hexanes & 1.9 & 2690.4 & \pm & 1903.9 & 121.5 & \pm & 79.5 & 2.6 & \pm & - \\
\hline Heptanes & 1.9 & 1238.1 & \pm & 834.8 & 72.1 & \pm & 36.6 & 14.3 & \pm & 12.1 \\
\hline 2,2,4-Trimethylpentane & 1.9 & 263.3 & \pm & - & 1893.4 & \pm & - & 614.6 & \pm & 487.2 \\
\hline Octanes & 1.9 & 1418.5 & \pm & 822.2 & 72.8 & \pm & 49.7 & 192.9 & \pm & 70.7 \\
\hline Nonanes & 0.03 & 3.4 & \pm & - & 3.4 & \pm & - & 14.4 & \pm & 6.4 \\
\hline Decanes & 0.003 & 1.8 & \pm & - & 3.1 & \pm & - & 3.2 & \pm & 1.4 \\
\hline Undecanes & 0.012 & 1.6 & \pm & - & 1.7 & \pm & - & 2.2 & \pm & 0.5 \\
\hline Dodecanes & 0.002 & 0.7 & \pm & - & 0.5 & \pm & - & 1.0 & \pm & 0.2 \\
\hline Tridecanes & 0.002 & 0.2 & \pm & - & $<\mathrm{LOQ}$ & \pm & - & 0.3 & \pm & 0.1 \\
\hline Tetradecanes & 0.009 & 0.1 & \pm & - & 0.0 & \pm & - & 0.1 & \pm & 0.0 \\
\hline 1,3-Butadiene & 1.0 & 7.3 & \pm & - & 18.0 & \pm & - & 6.2 & \pm & 0.4 \\
\hline Benzene & 8.5 & 727.7 & \pm & - & 145.6 & \pm & - & 265.9 & \pm & 342.2 \\
\hline Toluene & 4.1 & 192.6 & \pm & - & 205.6 & \pm & - & 550.6 & \pm & 70.2 \\
\hline Ethylbenzene & 3.4 & 79.2 & \pm & - & 34.1 & \pm & - & 48.6 & \pm & 16.5 \\
\hline m,p-Xylene & 3.1 & 337.0 & \pm & - & 139.3 & \pm & - & 94.8 & \pm & 132.1 \\
\hline o-Xylene & 3.0 & 79.9 & \pm & - & 39.9 & \pm & - & 54.9 & \pm & 21.2 \\
\hline 1,2,4-Trimethyl benzene & 0.008 & 0.2 & \pm & - & 1.1 & \pm & - & 0.3 & \pm & 0.1 \\
\hline 2-Ethyl toluene & 0.003 & 0.1 & \pm & - & 0.2 & \pm & - & 0.1 & \pm & 0.1 \\
\hline Isopropyl Benzene & 0.003 & 0.4 & \pm & - & 0.5 & \pm & - & 1.2 & \pm & 0.7 \\
\hline Styrene & 2.8 & 3.7 & \pm & - & 3.5 & \pm & - & 3.5 & \pm & 0.0 \\
\hline Isopropylbenzene & 2.8 & 6.8 & \pm & - & 5.1 & \pm & - & 6.2 & \pm & 1.0 \\
\hline 4-Ethyltoluene & 1.9 & 8.6 & \pm & - & 11.6 & \pm & - & 21.1 & \pm & - \\
\hline n-Propylbenzene & 2.5 & 8.2 & \pm & - & 5.3 & \pm & - & 5.8 & \pm & 4.5 \\
\hline 1,3,5-trimethylbenzene & 2.5 & 13.0 & \pm & - & 8.4 & \pm & - & 5.8 & \pm & 2.7 \\
\hline tert-butylbenzene & 2.6 & 4.6 & \pm & - & $<\mathrm{LOQ}$ & \pm & - & 3.6 & \pm & - \\
\hline 1,2,4-Trimethylbenzene & 6.1 & 25.9 & \pm & - & 21.3 & \pm & - & 16.5 & \pm & 0.1 \\
\hline s-Butylbenzene & 1.5 & 7.3 & \pm & - & 5.5 & \pm & - & 7.6 & \pm & 2.2 \\
\hline p-Isopropyltoluene & 472.8 & 496.4 & \pm & - & $<$ LOQ & \pm & - & 474.5 & \pm & - \\
\hline n-butylbenzene & 10.8 & $<\mathrm{LOQ}$ & \pm & - & $<\mathrm{LOQ}$ & \pm & - & $<\mathrm{LOQ}$ & \pm & - \\
\hline Naphthalene & 6.1 & 42.0 & \pm & - & 55.8 & \pm & - & $<\mathrm{LOQ}$ & \pm & - \\
\hline
\end{tabular}


Table S9. PM mass ( $\mu \mathrm{g} /$ filter) collected for different toxicity assays

\begin{tabular}{|c|c|c|c|c|}
\hline Particle mass $(\mu \mathrm{g})$ per filter & CNG test & RNG1 test & RNG2 test & Blank \\
\hline ROS assay & 17.3 & 9.17 & 8.25 & 0.68 \\
\hline Mutagenicity & 31.9 & 16.9 & 15.2 & 1.26 \\
\hline Molecular marker assays & 31.9 & 16.9 & 15.2 & 1.26 \\
\hline
\end{tabular}

Table S10. Abundance (in peak area) of compounds detected with XAD + GC/MS analysis from different exhaust samples

\begin{tabular}{|c|c|c|c|c|}
\hline Metabolite name & Retention time for unknowns (min) & CNG & RNG1 & RNG2 \\
\hline \multicolumn{2}{|c|}{ 2-Pentene, 2,4,4-trimethyl- } & $0.00 \mathrm{E}+00$ & $2.46 \mathrm{E}+05$ & $2.58 \mathrm{E}+05$ \\
\hline \multicolumn{2}{|c|}{ 3-Pentanol, 3-methyl- } & $1.05 \mathrm{E}+06$ & $1.06 \mathrm{E}+06$ & $0.00 \mathrm{E}+00$ \\
\hline \multicolumn{2}{|c|}{ n-Propyl acrylate } & $0.00 \mathrm{E}+00$ & $0.00 \mathrm{E}+00$ & $6.81 \mathrm{E}+06$ \\
\hline \multicolumn{2}{|c|}{ Cyclotrisiloxane, hexamethyl- } & $4.20 \mathrm{E}+05$ & $7.32 \mathrm{E}+04$ & $2.04 \mathrm{E}+07$ \\
\hline \multicolumn{2}{|c|}{ 3-Furaldehyde } & $8.40 \mathrm{E}+02$ & $9.20 \mathrm{E}+02$ & $6.13 \mathrm{E}+02$ \\
\hline \multicolumn{2}{|c|}{ Benzene, 1-chloro-4-(trifluoromethyl)- } & $4.17 \mathrm{E}+05$ & $9.82 \mathrm{E}+04$ & $3.90 \mathrm{E}+04$ \\
\hline \multicolumn{2}{|c|}{ 2,4-Octadiyne } & $0.00 \mathrm{E}+00$ & $0.00 \mathrm{E}+00$ & $0.00 \mathrm{E}+00$ \\
\hline \multicolumn{2}{|c|}{ Bicyclo[3.1.0]hex-2-ene, 2-methyl-5-(1-methylethyl)- } & $1.35 \mathrm{E}+05$ & $7.04 \mathrm{E}+04$ & $5.65 \mathrm{E}+04$ \\
\hline \multicolumn{2}{|c|}{ 2-Butenoic acid, 1-methylpropyl ester, (E)- } & $3.35 \mathrm{E}+04$ & $0.00 \mathrm{E}+00$ & $0.00 \mathrm{E}+00$ \\
\hline \multicolumn{2}{|c|}{ Santolina triene } & $2.21 \mathrm{E}+04$ & $1.38 \mathrm{E}+05$ & $1.43 \mathrm{E}+04$ \\
\hline \multicolumn{2}{|c|}{ Benzene, 1,3-dichloro- } & $3.74 \mathrm{E}+04$ & $2.10 \mathrm{E}+04$ & $1.34 \mathrm{E}+04$ \\
\hline \multicolumn{2}{|c|}{ 3-Carene } & $1.09 \mathrm{E}+05$ & $5.77 \mathrm{E}+04$ & $7.62 \mathrm{E}+04$ \\
\hline \multicolumn{2}{|c|}{ 7-Octen-4-one, 2,6-dimethyl- } & $1.22 \mathrm{E}+03$ & $9.70 \mathrm{E}+03$ & $1.28 \mathrm{E}+03$ \\
\hline \multicolumn{2}{|l|}{ p-Cresol } & $3.11 \mathrm{E}+04$ & $0.00 \mathrm{E}+00$ & $0.00 \mathrm{E}+00$ \\
\hline \multicolumn{2}{|c|}{ Benzene, 2-ethyl-1,4-dimethyl- } & $3.63 \mathrm{E}+04$ & $1.49 \mathrm{E}+04$ & $1.10 \mathrm{E}+04$ \\
\hline \multicolumn{2}{|c|}{ Indan, 1-methyl- } & $0.00 \mathrm{E}+00$ & $0.00 \mathrm{E}+00$ & $0.00 \mathrm{E}+00$ \\
\hline \multicolumn{2}{|c|}{ Benzene, 2-ethenyl-1,4-dimethyl- } & $3.00 \mathrm{E}+04$ & $1.74 \mathrm{E}+04$ & $1.02 \mathrm{E}+06$ \\
\hline \multicolumn{2}{|c|}{ Benzaldehyde, 4-ethyl- } & $8.39 \mathrm{E}+03$ & $0.00 \mathrm{E}+00$ & $0.00 \mathrm{E}+00$ \\
\hline \multicolumn{2}{|c|}{ Naphthalene } & $6.32 \mathrm{E}+05$ & $2.04 \mathrm{E}+05$ & $1.82 \mathrm{E}+05$ \\
\hline \multicolumn{2}{|c|}{ 1,3-Butadiene, 1,1,2,3,4,4-hexachloro- } & $5.46 \mathrm{E}+01$ & $5.00 \mathrm{E}+01$ & $5.84 \mathrm{E}+01$ \\
\hline \multicolumn{2}{|c|}{ 1,2-Benzisothiazole } & $2.28 \mathrm{E}+05$ & $1.14 \mathrm{E}+05$ & $1.04 \mathrm{E}+05$ \\
\hline \multicolumn{2}{|c|}{ Naphthalene, 2-methyl- } & $4.70 \mathrm{E}+04$ & $2.35 \mathrm{E}+04$ & $1.98 \mathrm{E}+04$ \\
\hline \multicolumn{2}{|c|}{ 2-Propenoyl chloride, 3-phenyl-, (E)- } & $1.03 \mathrm{E}+04$ & $9.63 \mathrm{E}+04$ & $0.00 \mathrm{E}+00$ \\
\hline \multicolumn{2}{|l|}{ 1-Octanol, 2-butyl- } & $2.73 \mathrm{E}+04$ & $1.00 \mathrm{E}+04$ & $1.15 \mathrm{E}+04$ \\
\hline \multicolumn{2}{|c|}{ Quinoline, 2-methyl- } & $9.79 \mathrm{E}+02$ & $8.19 \mathrm{E}+02$ & $0.00 \mathrm{E}+00$ \\
\hline \multicolumn{2}{|c|}{ Naphthalene, 1-methyl- } & $3.24 \mathrm{E}+04$ & $1.58 \mathrm{E}+04$ & $1.25 \mathrm{E}+04$ \\
\hline \multicolumn{2}{|c|}{ 2,5-Dimethylhexane-2,5-dihydroperoxide } & $1.19 \mathrm{E}+06$ & $5.32 \mathrm{E}+05$ & $4.80 \mathrm{E}+03$ \\
\hline \multicolumn{2}{|c|}{ 4-(2-Methylcyclohex-1-enyl)-but-2-enal } & $3.82 \mathrm{E}+05$ & $0.00 \mathrm{E}+00$ & $4.49 \mathrm{E}+03$ \\
\hline \multicolumn{2}{|c|}{ Acenaphthylene } & $4.16 \mathrm{E}+03$ & $1.43 \mathrm{E}+03$ & $2.36 \mathrm{E}+03$ \\
\hline \multicolumn{2}{|l|}{ Dimethyl phthalate } & $1.92 \mathrm{E}+04$ & $7.55 \mathrm{E}+03$ & $1.03 \mathrm{E}+04$ \\
\hline Dibenzofuran & & $2.94 \mathrm{E}+04$ & $1.58 \mathrm{E}+04$ & $1.14 \mathrm{E}+04$ \\
\hline Fluorene & & $6.15 \mathrm{E}+03$ & $0.00 \mathrm{E}+00$ & $0.00 \mathrm{E}+00$ \\
\hline
\end{tabular}




\begin{tabular}{|c|c|c|c|c|}
\hline \multicolumn{2}{|c|}{ 1-Pentadecene, 2-methyl- } & $5.62 \mathrm{E}+04$ & $2.30 \mathrm{E}+04$ & $2.58 \mathrm{E}+04$ \\
\hline \multicolumn{2}{|c|}{ Benzene, 1-bromo-4-phenoxy- } & $0.00 \mathrm{E}+00$ & $0.00 \mathrm{E}+00$ & $1.22 \mathrm{E}+02$ \\
\hline \multicolumn{2}{|c|}{ Dibenzothiophene } & $3.94 \mathrm{E}+03$ & $1.37 \mathrm{E}+02$ & $2.93 \mathrm{E}+03$ \\
\hline \multicolumn{2}{|c|}{ Anthracene } & $1.52 \mathrm{E}+04$ & $7.85 \mathrm{E}+03$ & $1.00 \mathrm{E}+04$ \\
\hline \multicolumn{2}{|c|}{ Dodecane, 4-cyclohexyl- } & $2.83 \mathrm{E}+04$ & $2.08 \mathrm{E}+04$ & $1.81 \mathrm{E}+04$ \\
\hline \multicolumn{2}{|c|}{ 2-(Pentyloxycarbonyl)benzoic acid } & $3.15 \mathrm{E}+04$ & $2.67 \mathrm{E}+04$ & $1.50 \mathrm{E}+04$ \\
\hline \multicolumn{2}{|c|}{ Phthalic acid, isobutyl undec-2-en-1-yl ester } & $0.00 \mathrm{E}+00$ & $0.00 \mathrm{E}+00$ & $0.00 \mathrm{E}+00$ \\
\hline Unknown & 4.961 & $3.65 \mathrm{E}+06$ & $1.83 \mathrm{E}+07$ & $1.63 \mathrm{E}+07$ \\
\hline Unknown & 5.299 & $0.00 \mathrm{E}+00$ & $3.64 \mathrm{E}+06$ & $3.56 \mathrm{E}+06$ \\
\hline Unknown & 5.335 & $0.00 \mathrm{E}+00$ & $1.05 \mathrm{E}+05$ & $0.00 \mathrm{E}+00$ \\
\hline Unknown & 5.448 & $0.00 \mathrm{E}+00$ & $1.05 \mathrm{E}+06$ & $0.00 \mathrm{E}+00$ \\
\hline Unknown & 5.746 & $5.61 \mathrm{E}+05$ & $4.50 \mathrm{E}+04$ & $2.89 \mathrm{E}+05$ \\
\hline Unknown & 6.593 & $1.33 \mathrm{E}+05$ & $3.19 \mathrm{E}+04$ & $3.77 \mathrm{E}+04$ \\
\hline Unknown & 6.69 & $0.00 \mathrm{E}+00$ & $0.00 \mathrm{E}+00$ & $0.00 \mathrm{E}+00$ \\
\hline Unknown & 6.934 & $0.00 \mathrm{E}+00$ & $0.00 \mathrm{E}+00$ & $1.10 \mathrm{E}+04$ \\
\hline Unknown & 7.107 & $0.00 \mathrm{E}+00$ & $0.00 \mathrm{E}+00$ & $1.87 \mathrm{E}+02$ \\
\hline Unknown & 7.674 & $0.00 \mathrm{E}+00$ & $5.43 \mathrm{E}+04$ & $5.69 \mathrm{E}+04$ \\
\hline Unknown & 10.244 & $4.71 \mathrm{E}+04$ & $3.29 \mathrm{E}+04$ & $3.18 \mathrm{E}+04$ \\
\hline Unknown & 10.539 & $0.00 \mathrm{E}+00$ & $0.00 \mathrm{E}+00$ & $9.96 \mathrm{E}+03$ \\
\hline Unknown & 10.796 & $0.00 \mathrm{E}+00$ & $0.00 \mathrm{E}+00$ & $3.08 \mathrm{E}+05$ \\
\hline Unknown & 11.781 & $2.21 \mathrm{E}+05$ & $1.17 \mathrm{E}+05$ & $1.03 \mathrm{E}+05$ \\
\hline Unknown & 12.696 & $0.00 \mathrm{E}+00$ & $0.00 \mathrm{E}+00$ & $1.29 \mathrm{E}+04$ \\
\hline Unknown & 12.918 & $4.66 \mathrm{E}+04$ & $2.78 \mathrm{E}+04$ & $2.75 \mathrm{E}+04$ \\
\hline Unknown & 14.249 & $0.00 \mathrm{E}+00$ & $1.65 \mathrm{E}+04$ & $1.60 \mathrm{E}+04$ \\
\hline Unknown & 14.254 & $5.21 \mathrm{E}+04$ & $3.68 \mathrm{E}+04$ & $2.26 \mathrm{E}+04$ \\
\hline Unknown & 14.369 & $6.43 \mathrm{E}+04$ & $3.86 \mathrm{E}+04$ & $1.50 \mathrm{E}+05$ \\
\hline Unknown & 15.277 & $1.90 \mathrm{E}+04$ & $1.94 \mathrm{E}+04$ & $1.21 \mathrm{E}+05$ \\
\hline Unknown & 17.738 & $9.42 \mathrm{E}+04$ & $8.01 \mathrm{E}+04$ & $7.24 \mathrm{E}+04$ \\
\hline Unknown & 18.741 & $1.06 \mathrm{E}+03$ & $1.05 \mathrm{E}+03$ & $1.40 \mathrm{E}+03$ \\
\hline Unknown & 18.743 & $7.65 \mathrm{E}+03$ & $2.84 \mathrm{E}+04$ & $2.19 \mathrm{E}+04$ \\
\hline Unknown & 20.175 & $2.62 \mathrm{E}+03$ & $0.00 \mathrm{E}+00$ & $0.00 \mathrm{E}+00$ \\
\hline Unknown & 33.34 & $3.87 \mathrm{E}+03$ & $9.22 \mathrm{E}+03$ & $0.00 \mathrm{E}+00$ \\
\hline Unknown & 33.44 & $2.39 \mathrm{E}+04$ & $1.40 \mathrm{E}+04$ & $9.05 \mathrm{E}+03$ \\
\hline Unknown & 43.883 & $9.23 \mathrm{E}+04$ & $4.81 \mathrm{E}+04$ & $9.43 \mathrm{E}+03$ \\
\hline Unknown & 76.454 & $1.98 \mathrm{E}+04$ & $2.83 \mathrm{E}+04$ & $1.04 \mathrm{E}+03$ \\
\hline Unknown & 76.623 & $0.00 \mathrm{E}+00$ & $0.00 \mathrm{E}+00$ & $0.00 \mathrm{E}+00$ \\
\hline
\end{tabular}




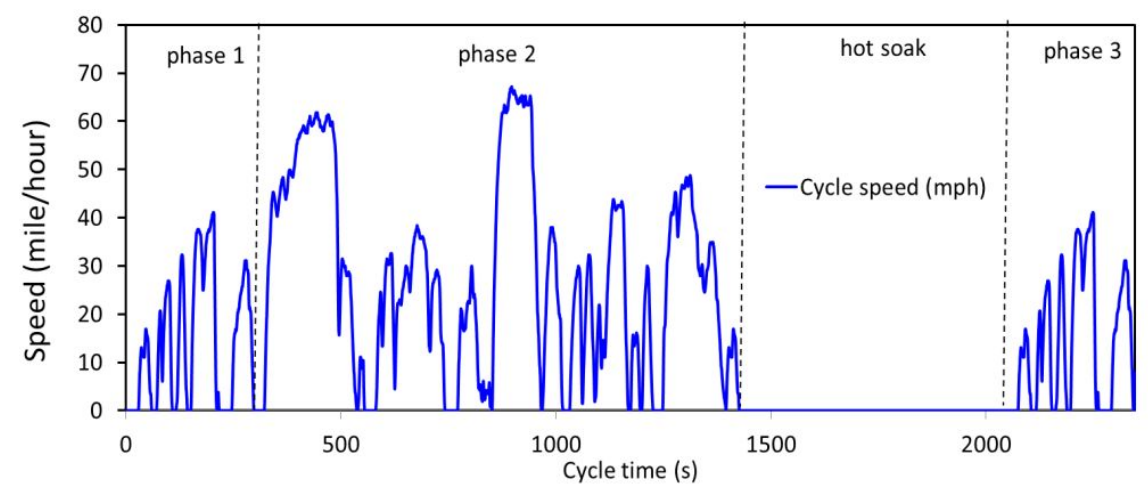

Figure S1. Speed time trace for the California Unified Cycle ${ }^{15}$
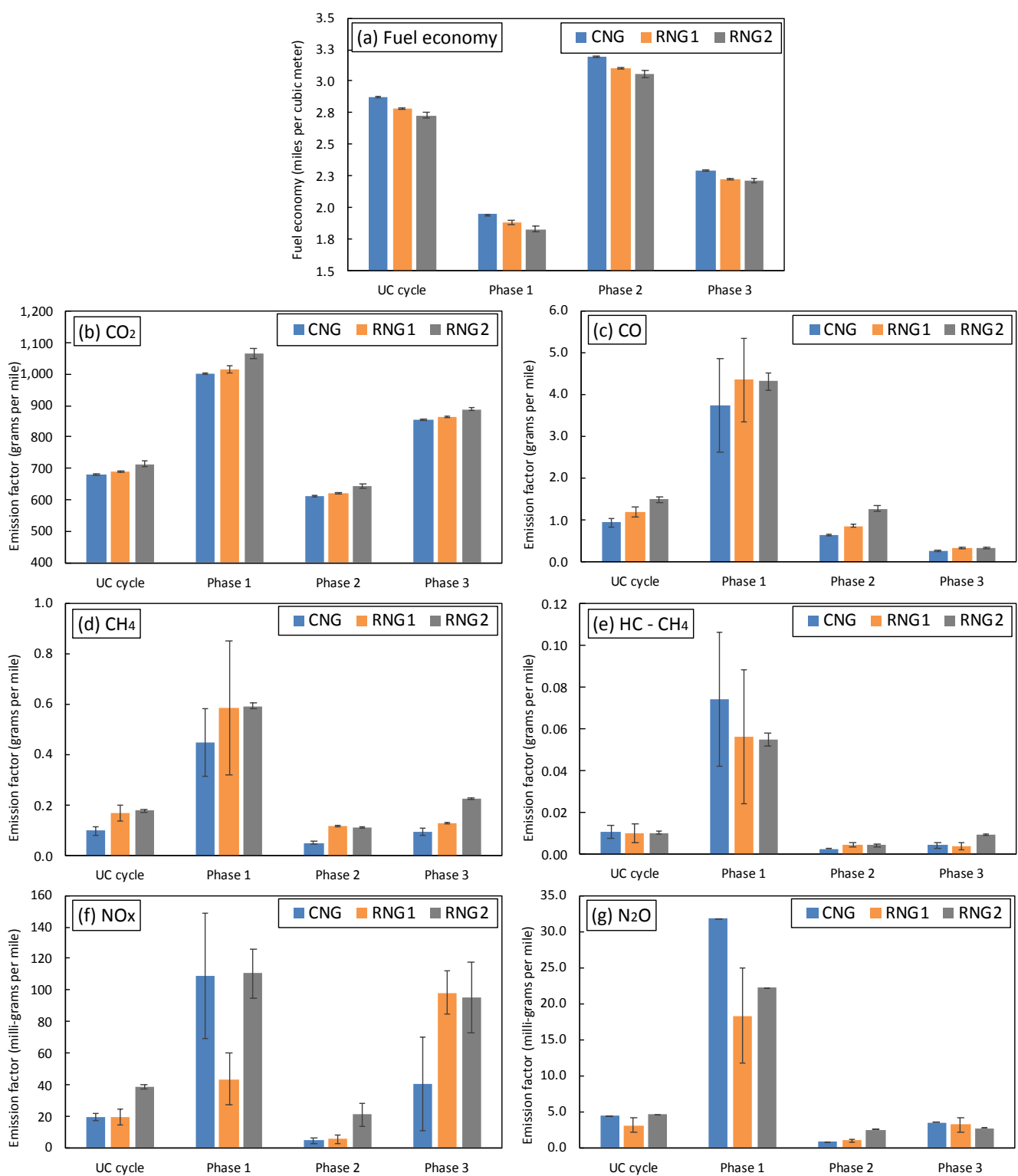

Figure S2. Fuel economy and emission factors of different pollutants for the averaged cold-start UC cycle as well as different phases of the cycle. Error bars define the range (min and max values) set by the duplicate tests. 

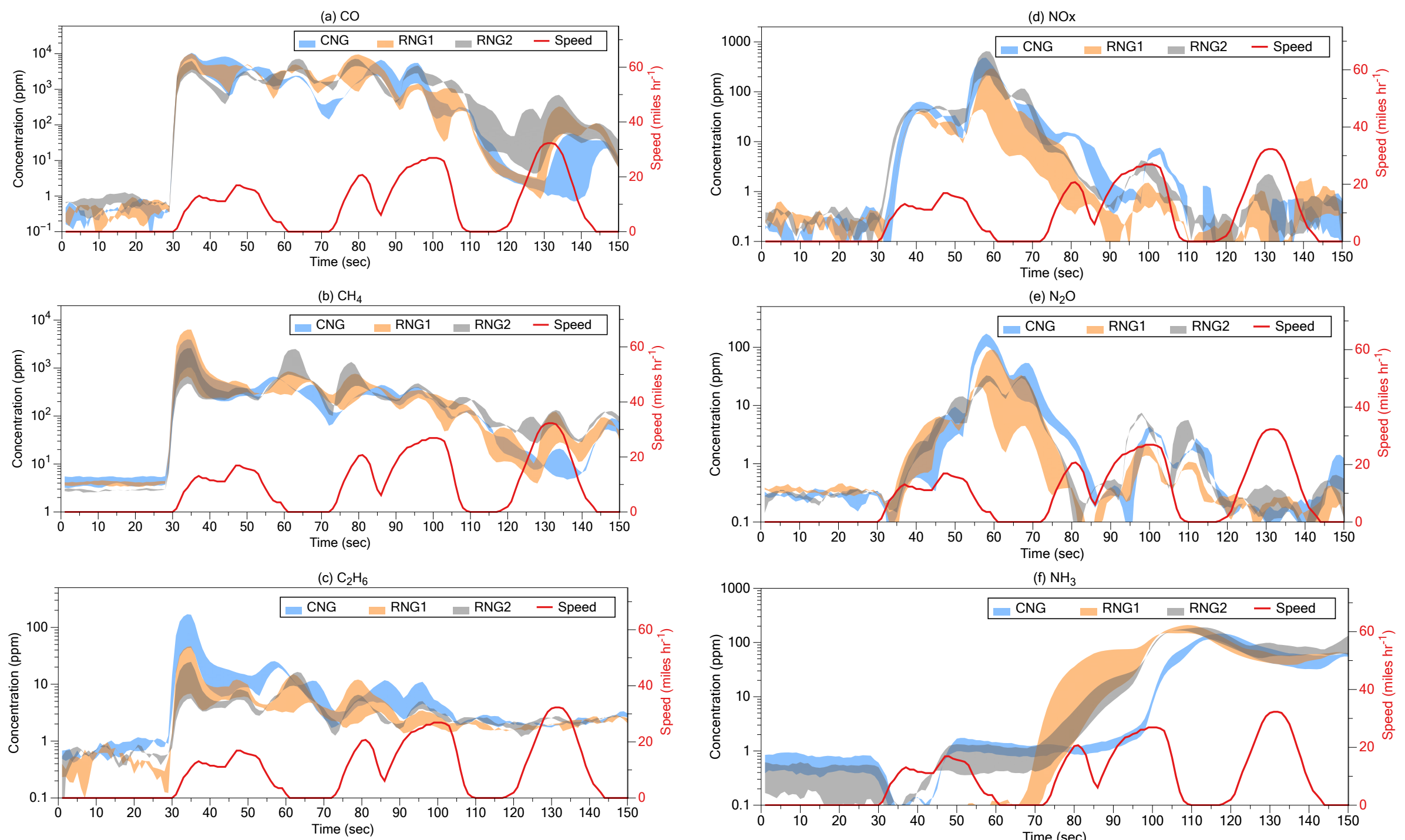

Figure S3 (a)-(f). Time series of concentration of different pollutants (ppm) measured by FTIR in the first 150 seconds of the cold-start UC cycle. The range for each fuel is outlined by the duplicate measurements from two cold-start UC cycle tests. 

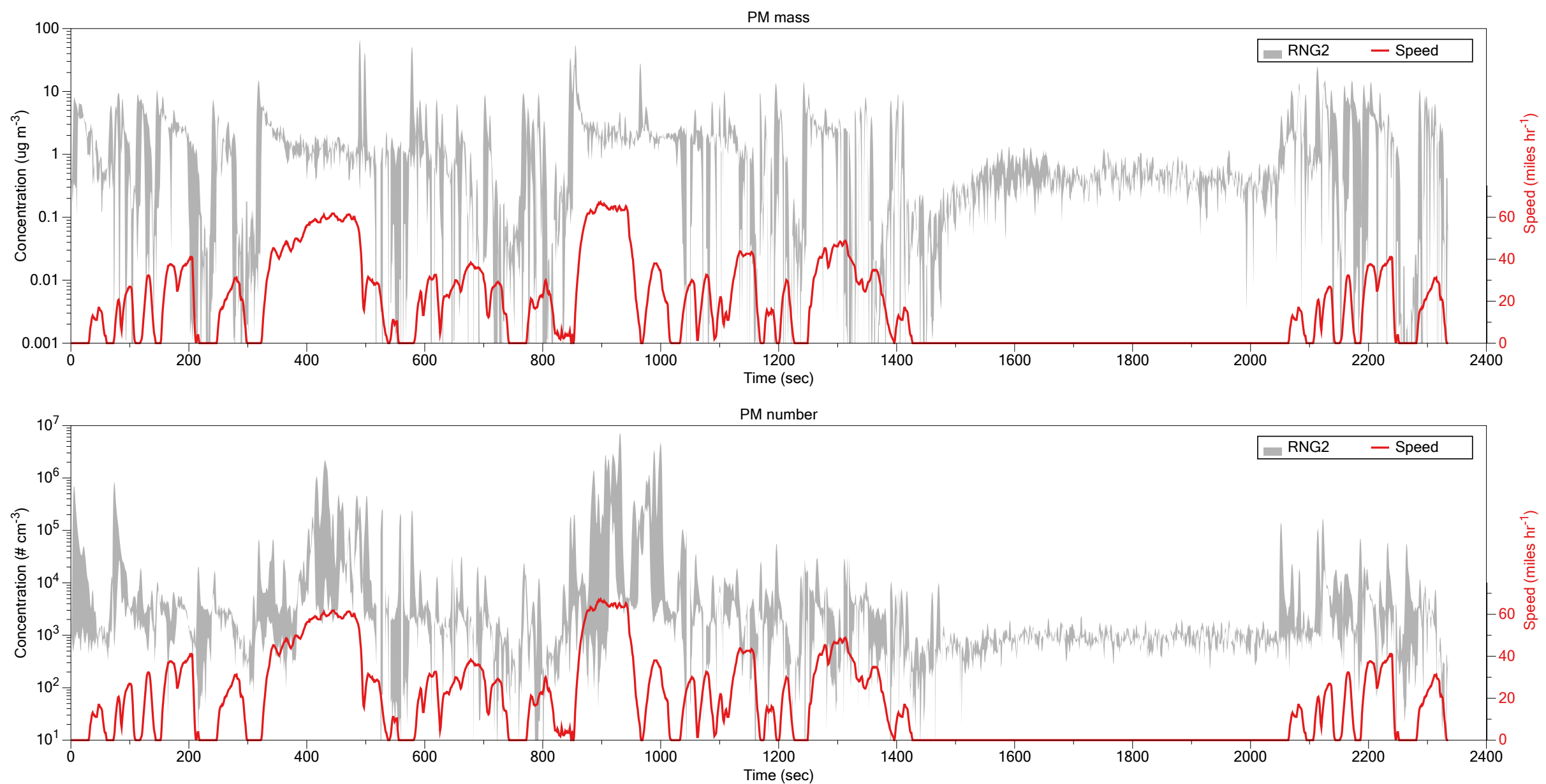

Figure S4 - Time series of particle mass concentration $\left(\mu \mathrm{g} \cdot \mathrm{m}^{-3}\right)$ and number concentration $\left(\# \cdot \mathrm{cm}^{-3}\right)$ measured from the CVS dilution tunnel. Showing the trace of RNG2 as an example 
(a) Ultrafine particle mass EF distribution

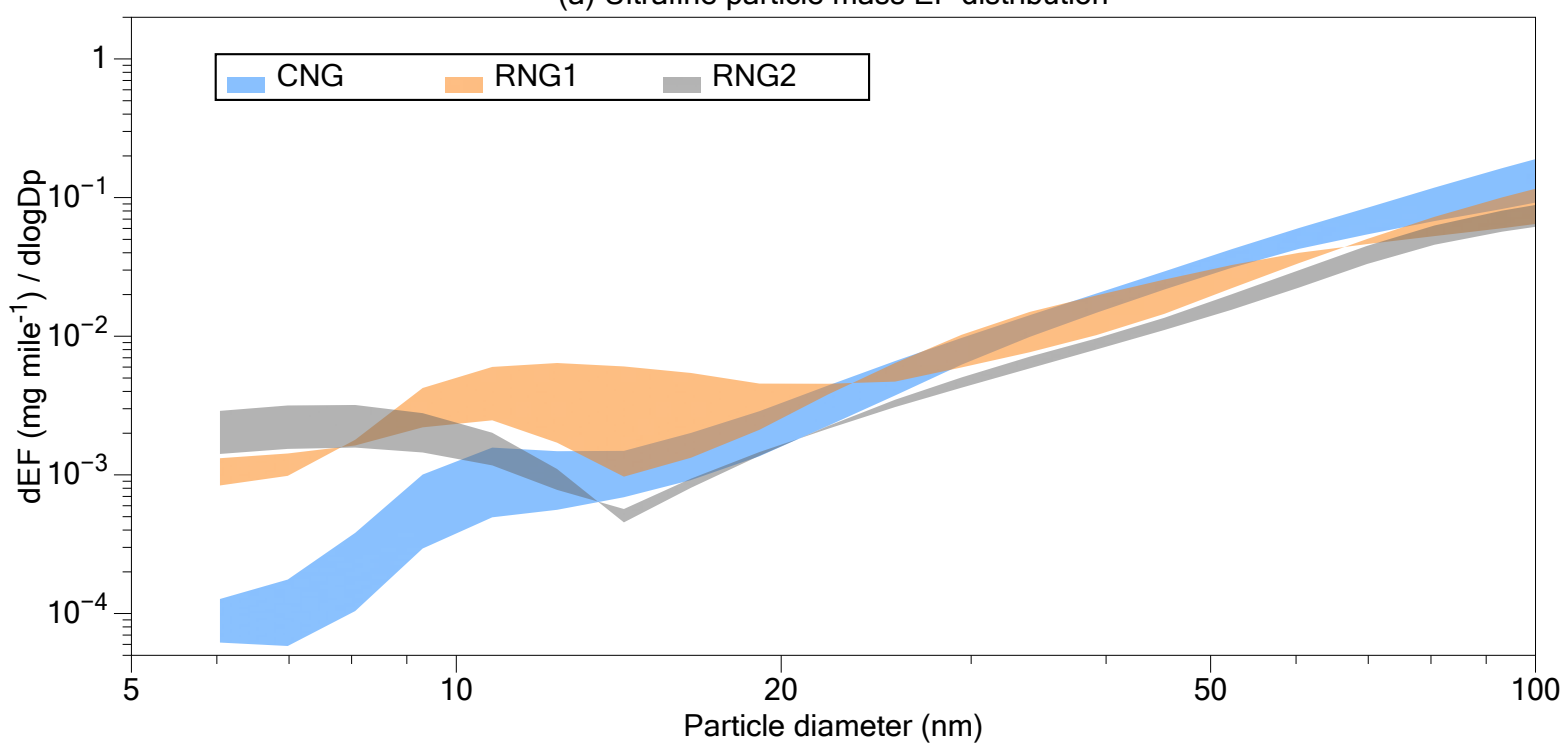

(b) Ultrafine particle number EF distribution

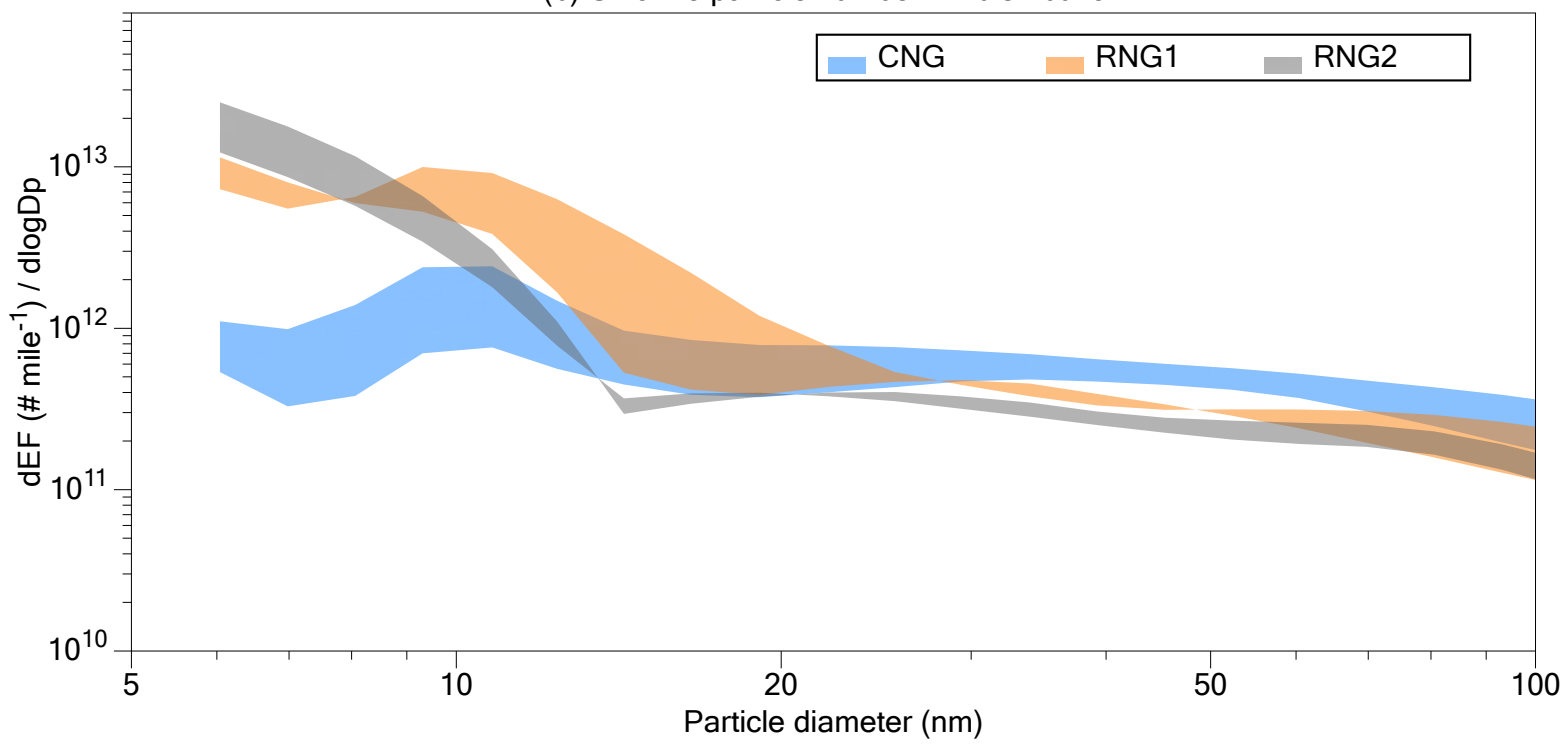

Figure S5. Distribution of (a) ultrafine particle mass emission factor (mg.mile $\left.{ }^{-1}\right)$ and (b) ultrafine particle number emission factor $\left(\# \cdot \mathrm{mile}^{-1}\right)$ from the tested vehicle running on different fuels. The range for each fuel (shaded area) is outlined by the duplicate measurements from two cold-start UC cycle tests. 
(a) Xylene - dark

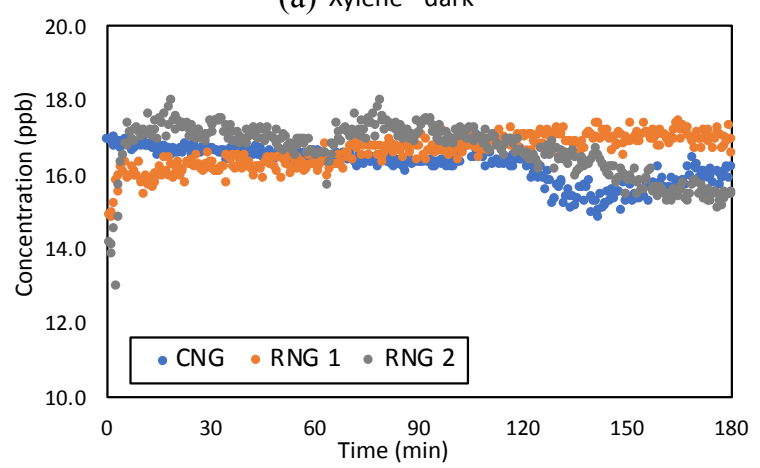

(c) Ethenone - dark

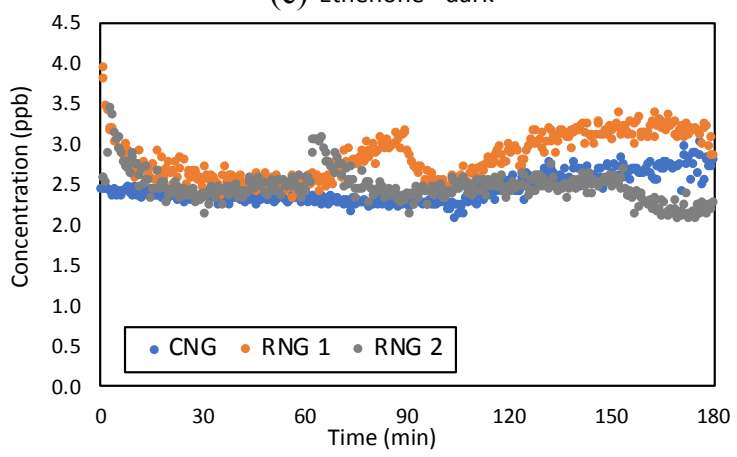

(b) Xylene - light

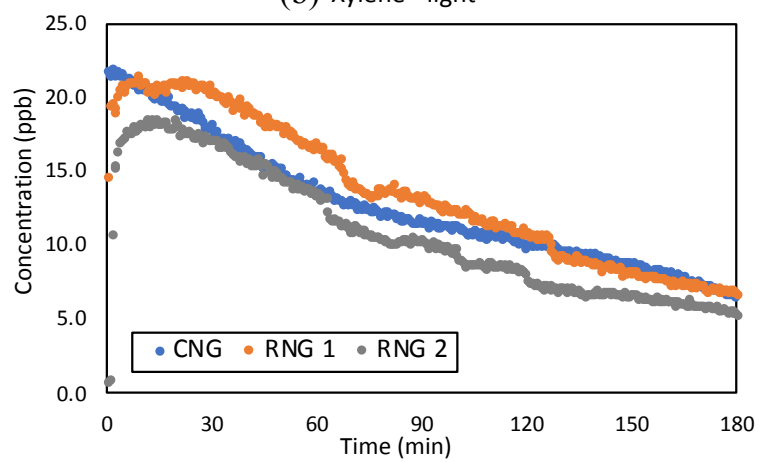

(d) Ethenone - light

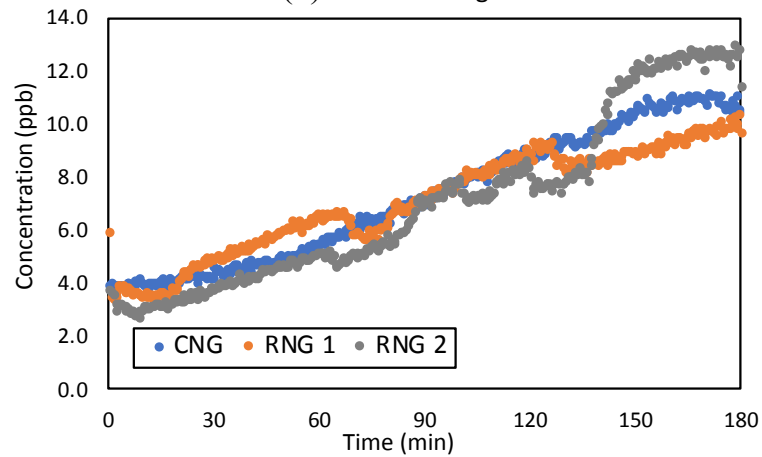

Figure S6. Typical concentration profiles (ppb) during atmospheric aging. (a) concentration of xylene during dark aging; (b) concentration of xylene during light aging; (c) concentration of ethenone during dark aging; (d) concentration of ethenone during light aging 
References:

(1) Li, Y.; Alaimo, C. P.; Kim, M.; Kado, N. Y.; Peppers, J.; Xue, J.; Wan, C.; Green, P. G.; Zhang, R.; Jenkins, B. M.; Vogel, C. F. A.; Wuertz, S.; Young, T. M.; Kleeman, M. J. Composition and Toxicity of Biogas Produced from Different Feedstocks in California. Environ. Sci. Technol. 2019. https://doi.org/10.1021/acs.est.9b03003.

(2) Peppers, J.; Li, Y.; Xue, J.; Chen, X.; Alaimo, C.; Wong, L.; Young, T.; Green, P. G.; Jenkins, B.; Zhang, R.; Kleeman, M. J. Performance Analysis of Membrane Separation for Upgrading Biogas to Biomethane at Small Scale Production Sites. Biomass and Bioenergy 2019, 128 , 105314. https://doi.org/10.1016/J.BIOMBIOE.2019.105314.

(3) Kado, N. Y.; Langley, D.; Eisenstadt, E. A Simple Modification of the Salmonella LiquidIncubation Assay Increased Sensitivity for Detecting Mutagens in Human Urine. Mutat. Res. Lett. 1983, 121 (1), 25-32. https://doi.org/10.1016/0165-7992(83)90082-9.

(4) Kado, N. Y.; Guirguis, G. N.; Flessel, C. P.; Chan, R. C.; Chang, K. -I; Wesolowski, J. J. Mutagenicity of Fine Airborne Particles: Diurnal Variation in Community Air Determined by a Salmonella Micro Preincubation (Microsuspension) Procedure. Environ. Mutagen. 1986, 8 (1), 53-66. https://doi.org/10.1002/em.2860080106.

(5) Zou, H.; Hastie, T. Regularization and Variable Selection via the Elastic Net. J. R. Stat. Soc. Ser. B Stat. Methodol. 2005, 67 (5), 768. https://doi.org/10.1111/j.1467-9868.2005.00527.x.

(6) Subramanian, K. A.; Mathad, V. C.; Vijay, V. K.; Subbarao, P. M. V. Comparative Evaluation of Emission and Fuel Economy of an Automotive Spark Ignition Vehicle Fuelled with Methane Enriched Biogas and CNG Using Chassis Dynamometer. Appl. Energy 2013, 105 (x), 17-29. https://doi.org/10.1016/j.apenergy.2012.12.011.

(7) Karavalakis, G.; Durbin, T. D.; Villela, M.; Miller, J. W. Air Pollutant Emissions of LightDuty Vehicles Operating on Various Natural Gas Compositions. J. Nat. Gas Sci. Eng. 2012, 4, 8-16. https://doi.org/10.1016/j.jngse.2011.08.005.

(8) Nevalainen, P.; Kinnunen, N. M.; Kirveslahti, A.; Kallinen, K.; Maunula, T.; Keenan, M.; Suvanto, M. Formation of NH3 and N2O in a Modern Natural Gas Three-Way Catalyst Designed for Heavy-Duty Vehicles: The Effects of Simulated Exhaust Gas Composition and Ageing. Appl. Catal. A Gen. 2018, 552 (September 2017), 30-37. https://doi.org/10.1016/j.apcata.2017.12.017.

(9) Behrentz, E.; Ling, R.; Rieger, P.; Winer, A. M. Measurements of Nitrous Oxide Emissions from Light-Duty Motor Vehicles: A Pilot Study. Atmos. Environ. 2004, 38 (26), 4291-4303. https://doi.org/10.1016/j.atmosenv.2004.04.027.

(10) Livingston, C.; Rieger, P.; Winer, A. Ammonia Emissions from a Representative In-Use Fleet of Light and Medium-Duty Vehicles in the California South Coast Air Basin. Atmos. Environ. 2009, 43 (21), 3326-3333. https://doi.org/10.1016/j.atmosenv.2009.04.009.

(11) Hajbabaei, M.; Karavalakis, G.; Johnson, K. C.; Lee, L.; Durbin, T. D. Impact of Natural Gas Fuel Composition on Criteria, Toxic, and Particle Emissions from Transit Buses Equipped with Lean Burn and Stoichiometric Engines. Energy 2013, 62, 425-434. https://doi.org/10.1016/j.energy.2013.09.040.

(12) Jayaratne, E. R.; He, C.; Ristovski, Z. D.; Morawska, L.; Johnson, G. R. A Comparative Investigation of Ultrafine Particle Number and Mass Emissions from a Fleet of On-Road Diesel and CNG Buses. Environ. Sci. Technol. 2008, 42 (17), 6736-6742. https://doi.org/10.1021/es800394x. 
(13) Lim, C.; Kim, D.; Song, C.; Kim, J.; Han, J.; Cha, J. S. Performance and Emission Characteristics of a Vehicle Fueled with Enriched Biogas and Natural Gases. Appl. Energy 2015, 139 (2015), 17-29. https://doi.org/10.1016/j.apenergy.2014.10.084.

(14) Thiruvengadam, A.; Besch, M. C.; Yoon, S.; Collins, J.; Kappanna, H.; Carder, D. K.; Ayala, A.; Herner, J.; Gautam, M. Characterization of Particulate Matter Emissions from a Current Technology Natural Gas Engine. Environ. Sci. Technol. 2014, 48 (14), 8235-8242. https://doi.org/10.1021/es5005973.

(15) Xue, J.; Li, Y.; Peppers, J.; Wan, C.; Kado, N. Y.; Green, P. G.; Young, T. M.; Kleeman, M. J. Ultrafine Particle Emissions from Natural Gas, Biogas, and Biomethane Combustion. Environ. Sci. Technol. 2018, 52 (22), 13619-13628. https://doi.org/10.1021/acs.est.8b04170.

(16) Amirante, R.; Distaso, E.; Tamburrano, P.; Reitz, R. D. Measured and Predicted Soot Particle Emissions from Natural Gas Engines. SAE Tech. Pap. 2015, 2015.

https://doi.org/10.4271/2015-24-2518. 